\title{
L'implantation des habitations circulaires en Bretagne et en Normandie à l'âge du Fer : un échange d'hommes et de savoirs
}

The construction of roundhouses in Brittany and Normandy during the Iron Age: a population change and a knowledge transfer La implementación de las casas circulares en Bretaña y Normandía a la Edad de Hierro: un intercambio de personas y conocimientos

\section{Céline Godard}

\section{OpenEdition}

Journals

Édition électronique

URL : http://journals.openedition.org/rao/2124

DOI : $10.4000 /$ rao. 2124

ISBN : 978-2-7535-3432-2

ISSN : 1775-3732

Éditeur

Presses universitaires de Rennes

\section{Édition imprimée}

Date de publication : 25 décembre 2013

Pagination : 165-186

ISBN : 978-2-7535-3430-8

ISSN : 0767-709X

Référence électronique

Céline Godard, «L'implantation des habitations circulaires en Bretagne et en Normandie à l'âge du Fer : un échange d'hommes et de savoirs », Revue archéologique de l'Ouest [En ligne], 30 | 2013, mis en ligne le 25 décembre 2015, consulté le 04 décembre 2020. URL : http://journals.openedition.org/rao/ 2124 ; DOI : https://doi.org/10.4000/rao.2124

Ce document a été généré automatiquement le 4 décembre 2020.

Tous droits réservés 


\title{
L'implantation des habitations circulaires en Bretagne et en Normandie à l'âge du Fer : un échange d'hommes et de savoirs
}

\author{
The construction of roundhouses in Brittany and Normandy during the Iron Age: \\ a population change and a knowledge transfer \\ La implementación de las casas circulares en Bretaña y Normandía a la Edad de \\ Hierro: un intercambio de personas y conocimientos
}

\section{Céline Godard}

1 Il y a peu de temps encore, l'habitat circulaire était considéré comme le mode de construction exclusif dans les îles Britanniques pour les périodes protohistoriques, tandis que sur le continent, la tradition architecturale s'incarnait dans des bâtiments sur poteaux quadrangulaires, à une ou deux nefs (Audouze et Buschsenshutz, 1989). Ce type d'habitat est d'ailleurs devenu si emblématique des îles Anglo-Saxonnes notamment depuis les fouilles de Little Woodbury en 1938 par G. Bersu - qu'il est considéré par certains auteurs, tel Hodson, comme un fossile directeur de l'âge du Fer en Grande-Bretagne (Harding, 2009).

2 Pourtant, les sources écrites antiques attestent de la présence de ces constructions sur notre territoire, comme l'illustre la description de Strabon: "Les Gaulois se construisent de grandes maisons de forme circulaire, en planches et en claies, et les recouvrent d'un épais toit de chaume " (Geographie, IV, 4, 3) ${ }^{1}$. De son côté, Jules César relate dans la Guerre des Gaules (Guerre des Gaules, V, 12) que les maisons des peuples bretons sont très semblables à celles des Gaulois, semant de ce fait un peu plus le trouble sur l'identité et la forme des habitations des peuples de l'Ouest.

Or, l'essor des grands décapages préventifs des trente dernières années a permis la découverte de plusieurs de ces bâtiments sur notre sol, notamment dans le Nord-Ouest de la France, relançant de ce fait la question de la présence et de l'origine de ce mode 
de construction : s'agit-il d'un savoir-faire hérité des îles Britanniques, d'une pratique architecturale développée en "vase clos » (selon l'expression d'Yvan Jahier in Jahier et al., 1997) ou encore des vestiges d'une culture commune, qui se serait étendue du nord de la Manche à la péninsule ibérique?

Le système économique implanté dès l'âge du Bronze et basé sur l'échange et la demande de matières premières (bronze, cuivre, ambre, etc.) a permis la mise en place de liens commerciaux et culturels très forts entre le continent et les îles Britanniques ; la position excentrée de ces dernières ne les empêchaient nullement de participer au grand commerce de l'Ouest de l'Europe, ni de nouer des relations privilégiées avec les populations voisines qui pourraient s'incarner sous cette forme architecturale circulaire.

5 Ainsi, fort d'un inventaire réactualisé des maisons circulaires de l'âge du Fer découvertes en Bretagne et en Normandie, nous tenterons ici d'éclairer les différents phénomènes qui ont pu conduire à la mise en place de ces constructions sur le sol français et de replacer ces dernières dans le contexte des relations trans-Manche à l'âge du Fer.

\section{L'architecture circulaire à la protohistoire sur les rives de la Manche}

6 Jusque dans les années quatre-vingt-dix, la différence majeure entre l'architecture britannique et la construction continentale s'opérait, pour les archéologues et les historiens, au niveau de la forme générale de la structure : la première adoptait une forme arrondie, tandis que la seconde se basait sur un tracé anguleux (d'après Audouze et Buchsenschutz in Jahier et al., 1997). Les découvertes de plus en plus nombreuses de ces maisons circulaires nous incitent désormais à vouloir pousser la réflexion au-delà des classifications préalablement établies, afin de mieux connaître ces habitations.

\section{Des îles Britanniques au continent européen à travers l'âge du Bronze et du Fer : une brève revue des structures d'habitat}

7 Si les récentes études ont démontré que la Grande-Bretagne n'avait plus le monopole de ce type de constructions (Dechezleprêtre et Ginoux, 2005; Marcigny et Talon, 2009), elle n'en reste pas moins considérée comme le berceau de cette tradition architecturale, grâce à la densité et la diversité de ses formes d'habitats circulaires ainsi que leur longévité sur ce territoire.

8 Les plus anciennes constructions circulaires retrouvées dans les îles Britanniques semblent être apparues à la transition du Néolithique et de l'âge du Bronze ancien, ou «Early Bronze Age» (période allant de 2000 à 1500 avant notre ère). En effet, des vestiges de maisons rondes, roundhouses, construites en pierre, ont été retrouvés sur des sites tels que Skara Brae à Orkney (nord de l'Écosse). Ces habitations, aux murs massifs, côtoient d'autres structures, de forme quadrangulaire cette fois-ci. Ces différents bâtiments disposent pour la plupart d'un foyer central et observent une organisation interne en cellule, annonçant les plans des wheelhouses (voir infra).

Ces premières constructions circulaires en pierre trouvent leur écho à l'extrémité sudouest de l'Angleterre, dans les Cornouailles - et plus précisément dans la partie nord de 
cette région - où les maisons rondes semblent s'être également implantées très tôt, dès le Campaniforme (Audouze et Buchsenschutz, 1989). Regroupées par vingtaine, elles forment des hameaux et sont constituées d'un mur de pierres imposant (environ un mètre de large), dont l'entrée peut se matérialiser par un simple trou dans le mur, un porche ou une pierre dressée ${ }^{2}$ ), à l'image des constructions de Bodmin Moor. Dans le sud des Cornouailles, la tradition architecturale est différente; en effet, les maisons sont cette fois-ci en bois, la structure étant basée sur des poteaux plantés, comme sur le site de Gwithian. Ce gisement a livré un bâtiment circulaire en bois de 4,5 mètres de diamètre, doté d'un poteau central, qui sera agrandi une cinquantaine d'années plus tard jusqu'à 7,6 mètres de diamètre, le poteau central ayant disparu au profit d'une structure reposant sur un double cercle de poteaux (Audouze et Buchsenschutz, 1989).

10 Le gisement de Black Patch (Sussex) fait figure de référence pour les gisements domestiques ruraux du milieu de l'âge du Bronze en Angleterre. En effet, il regroupe cinq maisons circulaires sur poteaux (Drewett, 1990), mesurant de cinq à sept mètres de diamètre et entourées de leurs annexes. Composées d'une ou de deux couronnes, elles associent aussi bien la construction sur poteau (pour la couronne interne) que sur tranchée (pour la couronne externe, illustrée par une paroi non porteuse). La maison circulaire en bois, de taille moyenne (cinq à huit mètres de diamètre), va alors dominer le Ier millénaire (Ralston et Pope, 2005 ; Webley, 2007). Parallèlement, les habitations en pierre se complexifient, se divisant à l'âge du Fer en plusieurs cellules disposées de façon rayonnante dans l'habitat, pour former le plan général d'une roue, d'où le nom de cette structure, wheelhouse.

11 Au premier âge du Fer, on assiste également à un phénomène de monumentalisation de l'habitat. En effet, celui-ci adopte des proportions imposantes: environ 15 mètres de diamètre, ce qui dégage une superficie de 176,71 mètres carrés au sol. Il s'implante la plupart du temps dans un enclos fossoyé circulaire (parfois doublé) en compagnie d'autres maisons en bois, comme à Pimperne Down (Dorset), ou à Little Woodbury (Wiltshire). C'est ce dernier site (fig. 1) qui a d'ailleurs donné l'impulsion à la recherche britannique sur les maisons circulaires dès le début des années quarante, sous la conduite de G. Bersu (1940). Toutefois, si cette habitation sur double cercle et porche est remarquablement bien organisée, elle ne témoignerait pas des constructions habituelles. En effet, pour certains auteurs, sa taille impressionnante suggèrerait la résidence d'élites locales, une construction communautaire ou encore un espace cultuel (Harding, 2009). La plus grande maison circulaire date toutefois du "Early Iron Age » (600-400 av. J.-C.) et se trouve sur le site de Bancroft (Buckinghamshire). Mesurant dixneuf mètres de diamètre, elle est constituée de trois couronnes (deux cercles de poteaux, le troisième étant fossoyé), illustrant une technique de construction élaborée et parfaitement maitrisée. 
Figure 1 : Maison circulaire sur double couronne de poteaux plantés de Little Woodbury, Angleterre. Figure 1 : Double-ring post roundhouse from Little Woodbury, England.



D’après Bersu, 1943, modifié. crannogs, ces structures circulaires formant des îlots artificiels (certaines étant construites sur pilotis) que l'on trouve principalement sur les lacs d'Irlande et d'Écosse. Si le plus vieux crannog recensé semble dater de la fin du Néolithique (Eilean Domhuill, sur le lac Olabhat en Écosse), la majorité d'entre eux remonterait à une période comprise entre 1000 et 500 avant J.-C. (Harding, 2009).

13 La maison circulaire suit les évolutions qui touchent le paysage rural britannique (Collis, 1990), s'insérant dans les hillforts (littéralement "collines fortifiées ») qui apparaissent à la fin de l'âge du Bronze - pour se développer durant l'âge du Fer - et renferment souvent plusieurs habitations ainsi que des structures de stockage. Lorsque ces lieux fortifiés sont progressivement abandonnés ou se transforment en centres régionaux imposants de plusieurs hectares, l'habitation ronde survit aux différents processus. Au $\mathrm{I}^{\mathrm{er}}$ siècle avant J.-C., le petit village de Glastonbury (Somerset) voit encore fonctionner en même temps quatorze maisons rondes dans son hameau.

n comparaison de l'incroyable longévité et de l'abondance de maisons circulaires, l'habitat quadrangulaire en Grande-Bretagne se trouve isolé et rare; il caractérise plutôt les structures de stockage ou autres annexes, ou bien des édifices à fonction cultuelle (Ralston et Pope, 2005), comme c'est le cas à Heathrow (Middlesex) ou Danebury (Hampshire). Selon Harding (2009), la transition vers la construction rectangulaire, forme déjà présente à l'âge du Fer de façon anecdotique et qui devient plus courante au $\mathrm{I}^{\mathrm{er}}$ siècle avant $\mathrm{J}$.-C., est progressive et ne dépend pas forcément de l'implantation de la culture romaine à la suite des conquêtes impériales. Parallèlement, la maison circulaire continue à être construite jusqu'au $v^{e}$ siècle de notre ère, 
notamment en périphérie des provinces romaines, comme l'attestent certaines structures d'Irlande et d'Écosse. Toutefois, quand ces habitations survivent, elles sont de plus petite taille et de forme moins régulière, ou bien sont au contraire de taille monumentale. Pour l'auteur, cette permanence de l'habitat circulaire serait une façon de proclamer une identité, un héritage indigène face au monde romain.

\section{En France, circulaire et quadrangulaire : une rencontre dès l'âge du Bronze}

15 L'habitat continental de l'âge du Fer nous est quant à lui bien mieux connu et a été abondamment traité tant sur sa forme que sur son organisation spatiale. Ainsi, la majorité des constructions sont basées sur un plan quadrangulaire ou carré, comportant une à deux nefs, et sont bâties en bois, sur poteaux plantés ou paroi porteuse (Dechezleprêtre et al., 1997a). Mais pour le Nord-Ouest de la France, l'habitat prend également une forme circulaire et ce, dès l'âge du Bronze, avec des sites importants tels Dampierre-sur-le-Doubs (Doubs), Roeux et Brebières (Pas-de-Calais), Erre et Seclin (Nord) ou encore le « clos du Lazaret » à Saint-Vaast-la-Hougue, situé sur l'île Tatihou dans la Manche (Marcigny et Ghesquière, 2003). À Malleville-sur-le-Bec, «Le buisson du Roui » (Eure), le gisement, daté du Bronze final, prend l'aspect d'un véritable village enclos, avec son enceinte monumentale de type ring-fort ainsi que vingt-quatre habitations circulaires sur poteaux, accompagnées de structures domestiques (greniers et fosses). Pour la Bretagne, les travaux de P.-R. Giot et J. Briard (Giot et al., 1995 ; Briard et al., 1988) ainsi que A. Duval (Duval et al., 1988) ont permis de mettre en évidence des unités d'habitations en pierre, de forme rectangulaire (comme à Brenillis, sur le site de "Goarem-ar-Poulenou ») ou circulaire à l'image de "La grosse Roche » à Saint-Jacut-de-la-Mer (Briard et al., 1988) ou du "Vivier» à Quiberon. Les constructions de l'Ouest de la France à l'âge du Bronze présentent ainsi un profil à rattacher à la fois aux habitations des îles Britanniques mais également aux traditions architecturales continentales.

16 Ailleurs en Europe tempérée, cette architecture circulaire est peu présente, à l'exception de certaines régions de la péninsule ibérique et du nord de la Manche (voir infra). Elle est cependant observée au sein d'espaces cultuels, à l'image des structures de Manching (Bavière) ou de Molesme (Côtes d'Or), posant ainsi la question de l'interprétation de ces bâtiments.

\section{Enclos, habitat ou temple ? Les problèmes de l'interprétation}

Les vestiges en creux des maisons circulaires renseignent peu sur l'élévation de ces structures et lorsque cette dernière peut être établie, il est difficile alors d'identifier avec certitude les activités qui pouvaient se dérouler dans ces bâtiments.

En effet, dans un premier temps, il est particulièrement malaisé de distinguer les enclos circulaires des habitations de même forme et l'on ne peut souvent se baser que sur des incohérences (absence de symétrie) ou bien sur la largeur et la profondeur des trous de pieux et de poteaux (lorsqu'il ne s'agit pas d'une construction sur tranchée, rendant la tâche encore plus ardue). On peut ainsi prendre l'exemple du site de Plessis-Gassot (Val d'Oise) où un double cercle de poteaux a été retrouvé, formant une structure de dixsept mètres de diamètre datant du second âge du Fer. Or, le plan très irrégulier joue en 
la défaveur d'une construction charpentée et la structure a donc été interprétée en tant qu'enclos (Dechezleprêtre et Ginoux, 2005).

De même, l'interprétation d'un bâtiment circulaire sur poteaux s'avère parfois difficile car ce dernier peut être rattaché à un espace cultuel ou funéraire. En effet, les structures circulaires peuvent également prendre part à ce type d'espace, comme l'illustre le site de Molesme "Sur les Creux ». Situé en Côte d'Or, dans les méandres d'une rivière, le site a été daté de La Tène finale. Plusieurs structures circulaires ont été mises au jour (Petit, 2003), s'organisant sur une grande esplanade quadrangulaire délimitée par un enclos. Le bâtiment principal, de 14,7 mètres de diamètre, est constitué d'une couronne de poteaux et de deux larges poteaux internes alignés. Il est entouré par quatre autres bâtiments dont trois circulaires et un quadrangulaire, chacun placé à un point cardinal (Petit, 2003). C'est dans l'édifice rectangulaire qu'a été retrouvé un abondant mobilier osseux (principalement de bovidés et de porcs) ainsi que des amphores de type Dressel I, semblant illustrer des pratiques de banquet et pouvant ainsi conférer au site une dimension cultuelle.

En Angleterre, ce type de site à fonction rituelle a également été mis au jour, même si aucun temple ou édifice cultuel au sens strict du terme n'a été retrouvé (Harding, 2009). Toutefois, le site de "Fison Way » à Thetford (Norfolk), présente une implantation à l'organisation presque similaire à celle de Molesme. En effet, les structures s'organisent sur une grande esplanade quadrangulaire d'une centaine de mètres de côté, dotée d'une entrée à l'est (Harding, 2009, p. 225). Un bâtiment de 13 mètres de diamètre sur poteaux, orienté à l'est, est accompagné d'enclos contenant des fosses. Deux nouvelles structures circulaires lui seront plus tard associées. Toutefois, contrairement à Molesme, le site n'a révélé aucune présence de dépôts votifs ; son interprétation en tant qu'espace rituel est principalement basée sur le contraste de son organisation avec les implantations domestiques britanniques habituelles.

21 Ces quelques exemples illustrent ainsi la difficulté d'interpréter les bâtiments circulaires, surtout lorsque ces derniers se situent sur des sites où ont eu lieu des banquets et des pratiques communautaires, voire cultuelles. Ainsi, comment différencier ces structures (même rares), pouvant abriter des activités rituelles, des habitations? En réalité, c'est le contexte du site qui apporte la plupart du temps la réponse à cette question. En effet, sans la découverte du mobilier lié aux banquets et des restes animaux déposés dans la construction quadrangulaire à Molesme, on aurait $\mathrm{pu}$ considérer ce gisement comme une occupation domestique, renfermant trois maisons rondes disposées autour d'une quatrième plus imposante, comme cela peut être le cas en Normandie avec les constructions de Poses sur le site "Sur la mare " (voir notre corpus), même si l'organisation de ce dernier est beaucoup moins bien structurée. La délicatesse de l'interprétation s'accentue encore en présence de bâtiments circulaires isolés, au mobilier faible voire absent.

22 Toutefois, Y. Menez (Menez et al., 1990) propose plusieurs critères qui pourraient aider à distinguer une occupation domestique des autres types de gisements pouvant prêter à confusion. Ainsi, il prend en compte la largeur de la porte du bâtiment (qui permettrait de voir si cette dernière est adaptée à taille d'homme ou d'animaux, et distinguer de cette façon l'habitat de l'enclos), la taille des poteaux (si ces derniers sont imposants, ils pourraient être révélateurs d'une charpente), la présence d'aménagements internes (un foyer par exemple, qui illustrerait un bâtiment à usage domestique) et enfin le contexte général, comme la présence ou l'absence de maisons pour jouer ce rôle aux 
alentours, la position de la structure par rapport à l'ensemble et la présence de mobiliers et d'annexes domestiques (greniers, fosses, puits, etc.). À l'aide de ces éléments, il est ainsi possible d'identifier et de différencier la plupart du temps les habitations circulaires des autres édifices de même forme mais à la fonction différente.

\section{Construire la maison circulaire : techniques, matériaux et organisation de l'espace interne}

\section{Matériaux et type de construction}

Les maisons circulaires peuvent être construites de différentes façons, en pierre ou en bois, selon la disponibilité des matériaux sur place. Si la pierre peut être utilisée de façon classique pour les soubassements (solin de pierre servant de support à une paroi en matériaux périssables) ou l'ensemble du bâtiment (maçonnerie en pierres sèches), le bois peut être employé de diverses manières. L'habitation peut ainsi prendre la forme d'une construction sur poteaux plantés ou bien d'un bâtiment sur fossé, à l'image des ring-groove houses ou bien des stake-wall houses britanniques. Le fossé des premières pouvait recevoir un assemblage de planches ou une paroi en pieux et clayonnages, illustrés de façon archéologique par un fossé au profil en U. Le second type d'habitation basée sur un fossé (fig. 2) s'illustre également par une tranchée de fondation mais, cette fois-ci, sa mise en œuvre est différente: de son profil en $\mathrm{V}$, les archéologues ont restitué une paroi composée de pieux serrés (Cunliffe, 2005), regroupés et attachés par un lien végétal au sommet pour former à la fois le mur et le toit, ou bien supportant une sablière haute qui servira de base à la charpente; ce type de structure ne comportera pas de porche. En ce qui concerne les maisons circulaires sur poteaux plantés, elles peuvent s'organiser sur un ou plusieurs cercles de pieux en bois et peuvent disposer d'un poteau central au cœur de la construction. Il apparaît cependant que ce poteau n'est pas indispensable au maintien de la structure (Ralston et Pope, 2005) et qu'il est le plus souvent utilisé pour soutenir la charpente lors de la mise en place de cette dernière. De plus, la présence d'un foyer central dans les constructions suggère l'absence de poteau central vertical. 
Figure 2 : Reconstitution d'une « stake-wall house » basée sur les plans des structures découvertes à Danebury, Angleterre.

Figure 2 : Stake-wall house reconstruction, based on Danebury structures, England.

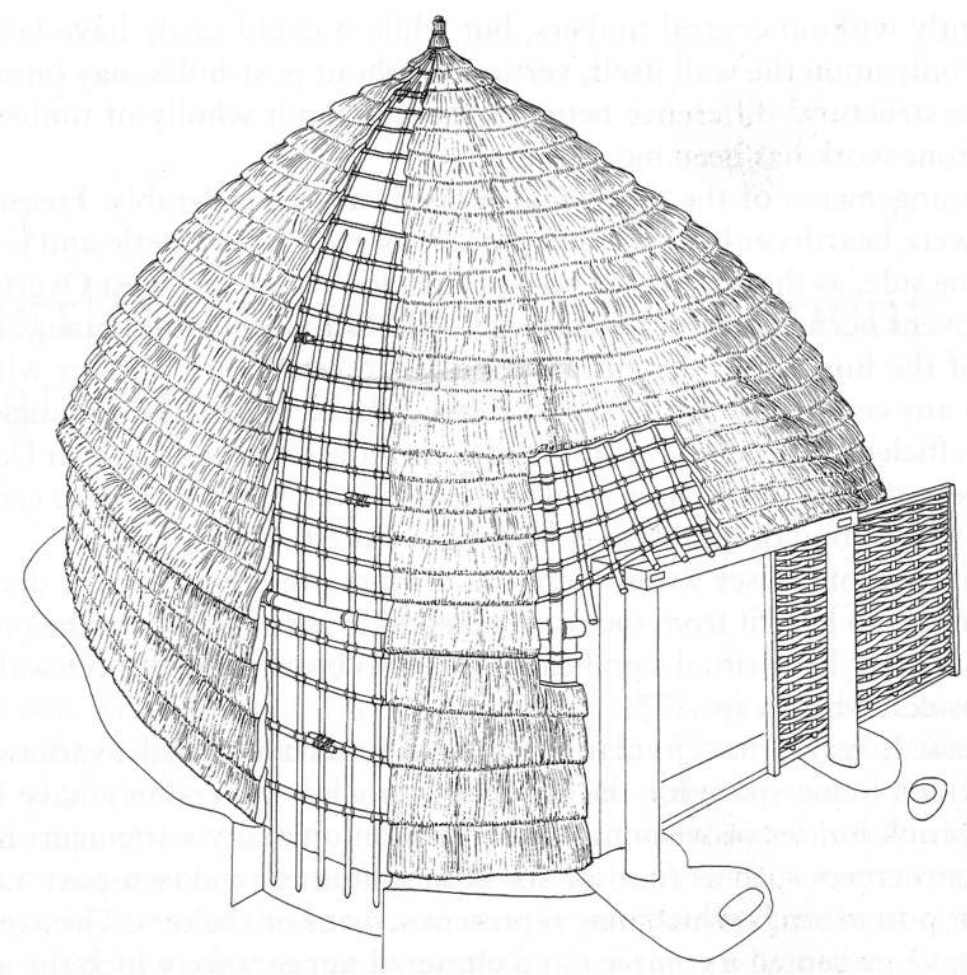

D'après Cunliffe, 2005 ; fig. nº 12.21.

Enfin, la paroi du bâtiment peut être réalisée en clayonnage et torchis, mais Strabon mentionne également la possibilité d'utiliser des planches et des claies (voir supra). L'utilisation du chaume ou de bardeaux de chêne est quant à elle attestée pour la couverture du toit (d'après Strabon, Géographie, IV, 4, 3 et Vitruve, De Architectura, II, 4). En ce qui concerne la charpente, les restitutions (Audouze et Buchsenschutz, 1989; Hodara, 2005) proposent un toit de forme conique basé sur des chevrons, ainsi qu'un renforcement de la structure au niveau de l'entrée, cette dernière brisant l'équilibre de la construction.

Dans les maisons à double couronne de poteaux, c'est le second cercle qui reçoit les chevrons du toit pendant que le premier les soutient. Ainsi, la couronne externe n'a pas besoin d'être profondément ancrée dans le sol (ce qui peut parfois expliquer le plan irrégulier voire manquant des vestiges des maisons à double couronne).

Enfin, la pente du toit est choisie en fonction des matériaux disponibles et des conditions climatiques locales (notamment l'enneigement) ; augmenter la pente du toit diminue sa pression. Ainsi, la pente idéale a été calculée à 45 degrés (Harding, 2009) de façon à ce que le poids ne porte pas seulement sur les entraits tout en assurant une bonne protection contre les éléments météorologiques. Un angle plus important serait de faible utilité ; en effet, augmenter la pente ne serait-ce que de 5 degrés ne libère en réalité que 0,10 mètre sur l'aire au sol (Pope, 2007). Or, l'aménagement de la maison circulaire est conçu de façon à optimiser au mieux l'espace interne et l'on peut imaginer l'ajout d'un étage pour obtenir plus d'espace de stockage, ou encore prendre 
pour exemple les ring-ditch houses dont le fossé interne à la construction permet la conservation de denrées.

\section{Orientation et organisation de l'espace interne}

L'orientation de l'habitation, déterminée par son entrée (pouvant être développée sous la forme d'un porche de deux, quatre ou plus rarement six poteaux), prend en compte la luminosité mais aussi le sens du vent dominant. En effet, R. Pope a récemment démontré (Ralston et Pope, 2005) que les fonctions symboliques que l'on attribuait à l'orientation ont finalement peu à voir avec la raison pratique de cette dernière, à savoir trouver la meilleure luminosité tout en obtenant le meilleur abri. C'est pour cette raison qu'une grande partie des maisons circulaires du nord des îles Britanniques sont orientées au sud-est, les façades ouest et est étant sources de courant d'air. L'étude effectuée par R. Pope détermine en effet que l'optimal entre l'abri et la lumière se situe entre le sud et le sud-est; cette donnée permet de comprendre l'organisation des activités domestiques, qui dépendent en grande partie de la luminosité naturelle.

En effet, la répartition du mobilier dans l'habitat semble indiquer que la partie arrière de la construction était réservée pour le couchage, son retranchement permettant de créer un espace privé tandis que la partie avant de l'habitation, plus ouvert à la circulation et plus lumineux, semble avoir été le lieu d'activités domestiques, comme en témoignent dans certaines constructions les vestiges de pesons de tissage. Pour les habitations à double couronne, il est possible que cette organisation ait pu se modifier, avec la mise en place de partitions internes, annulaires ou radiales. Les autres aires d'activités s'organisaient également autour du foyer, généralement centré. Ainsi disposé, ce dernier distribue la chaleur et la lumière dans toute l'habitation et réduit de cette façon les risques d'incendie.

Le stockage de denrées pouvait quant à lui s'effectuer à l'aide de céramiques, de fosses ou même de souterrains (Ralston et Pope, 2005). Mais l'étage de la construction a également pu jouer un rôle conséquent dans l'activité de stockage. En effet, dans une habitation circulaire établie sur deux cercles de poteaux, entre 20 et $30 \%$ du volume se trouve dans l'espace du toit; il est alors possible de restituer un étage car il semble peu probable que cet espace libre ait été perdu. R. Pope (Pope, 2007) remarque que les structures mesurant jusqu'à neuf mètres de diamètre peuvent être construites sans couronnes internes, mais il s'avère que beaucoup d'entre elles en possèdent une, notamment celles mesurant entre sept et neuf mètres de diamètre. Cet agencement, pourtant facultatif, pourrait servir de base à l'aménagement d'un étage. Toutefois, il est peu probable que ce dernier ait servi comme lieu de vie secondaire ; en effet, situé audessus du foyer, il devait être plutôt chaud et surtout enfumé (Pope, 2007). Il est ainsi interprété comme un espace de stockage supplémentaire, en particulier pour la nourriture qui, par la même occasion, était gardée au sec et désinfectée.

\section{L'apport de l'archéologie expérimentale}

Plusieurs expérimentations ont permis de mieux connaître les méthodes de construction de la maison circulaire, comme ce fut le cas avec le projet «Butser Ancient Farm » (fig. 3) mis en place en 1972 par P. J. Reynolds en Angleterre. La reconstitution de trois maisons circulaires (basées sur des plans de structures retrouvées en fouille, 
dont celle de Pimperne Down) et de leur environnement a permis d'observer la quantité de matériaux nécessaires à la mise en place de ces structures. Plus de deux cents arbres ont été nécessaires à la construction d'une seule construction de quinze mètres de diamètre, ainsi que sept tonnes de paille de blé pour la toiture en chaume. Elles ont également révélé des méthodes de construction judicieuses (l'utilisation expérimentale de linteaux pour rejoindre les poteaux de la couronne interne s'est révélée idéale, tout comme le fait de faire reposer la base des chevrons sur le sol pour une meilleure stabilité), tout en observant l'évolution des habitations dans le temps (Harding et al., 1993). Ainsi, la construction la plus imposante est restée en place durant un peu plus de seize ans, ne subissant que le remplacement du porche et d'une partie de la toiture, dans des conditions climatiques extrêmement difficiles. Ce type d'expérimentation fut reconduit sur de nombreux sites en Grande-Bretagne (Bascomb Copse, Chacewater, Castel Henllys, etc.), preuve de l'intérêt porté par la communauté scientifique à ces constructions ainsi que de la volonté de vulgarisation auprès du grand public. Ainsi, la maison circulaire est plus que jamais au centre des problématiques archéologiques concernant les constructions de l'âge du Fer et nous tenterons d'apporter ici notre modeste contribution à celles-ci, en réactualisant le corpus des habitations circulaires en Normandie et en Bretagne.

Figure 3 : Construction expérimentale basée sur les plans de la structure de Pimperne Down, sur le site de Butser Farm en Angleterre.

Figure 3 : Experimental reconstruction based on the plans of Pimperne Down roundhouse at Butser Farm, England.

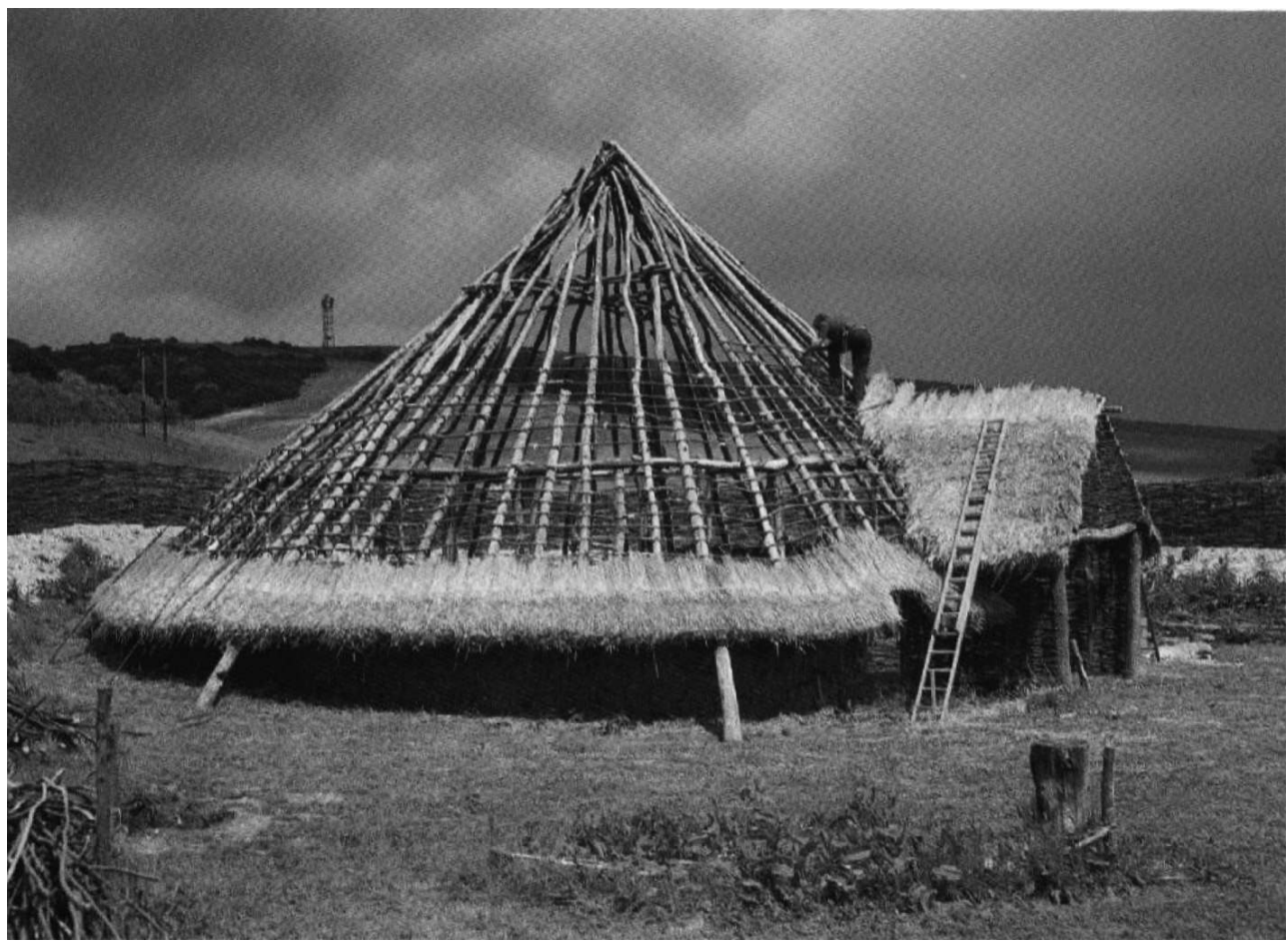

D'après Harding, 2009 


\section{L'habitat circulaire en Bretagne et en Normandie : inventaire, description et analyse des structures}

\section{Présentation du corpus}

31 Afin de répondre aux problématiques sur l'implantation de ces structures dans nos régions, nous avons réalisé un inventaire des sites d'habitats circulaires de l'âge du Fer en Bretagne et en Normandie (Godard, 2010), en nous basant sur les rapports de fouilles, la Carte archéologique ainsi que sur les travaux de T. Dechezleprêtre (1997a et b, 2005), I. Jahier (1997) et Y. Menez (1990) qui synthétisent dans leurs articles la découverte de ces structures par régions.

32 Ainsi, quinze sites ont été répertoriés (fig. 4), comportant chacun une ou plusieurs maisons circulaires, aussi bien sur poteaux plantés, sur tranchées de fondation ou en pierre. Nous n'avons retenu ici que les habitats ayant été identifiés en tant que tel, bien conscients des limites d'un tel inventaire, basé sur les connaissances actuelles des sites reconnus et fouillés. De plus, la difficulté de dater des sites parfois sans mobilier (n'indiquant ainsi qu'une période vaste et imprécise) nous a conduits à inclure dans notre étude les sites d'habitats circulaires à la transition du Bronze final et du premier âge du Fer (à l'image de Cahagnes ou de Cagny dans le Calvados) ainsi que ceux postérieurs à La Tène, comme Étaimpuis (Seine-Maritime), datés du $\mathrm{I}^{\mathrm{er}}$ siècle de notre ère. 
Figure 4 : Carte de répartition des sites comportant des maisons circulaires de l'âge du Fer en Bretagne et en Normandie.

Figure 4 : Location map of Iron Age roundhouse sites in Brittany and Normandy.

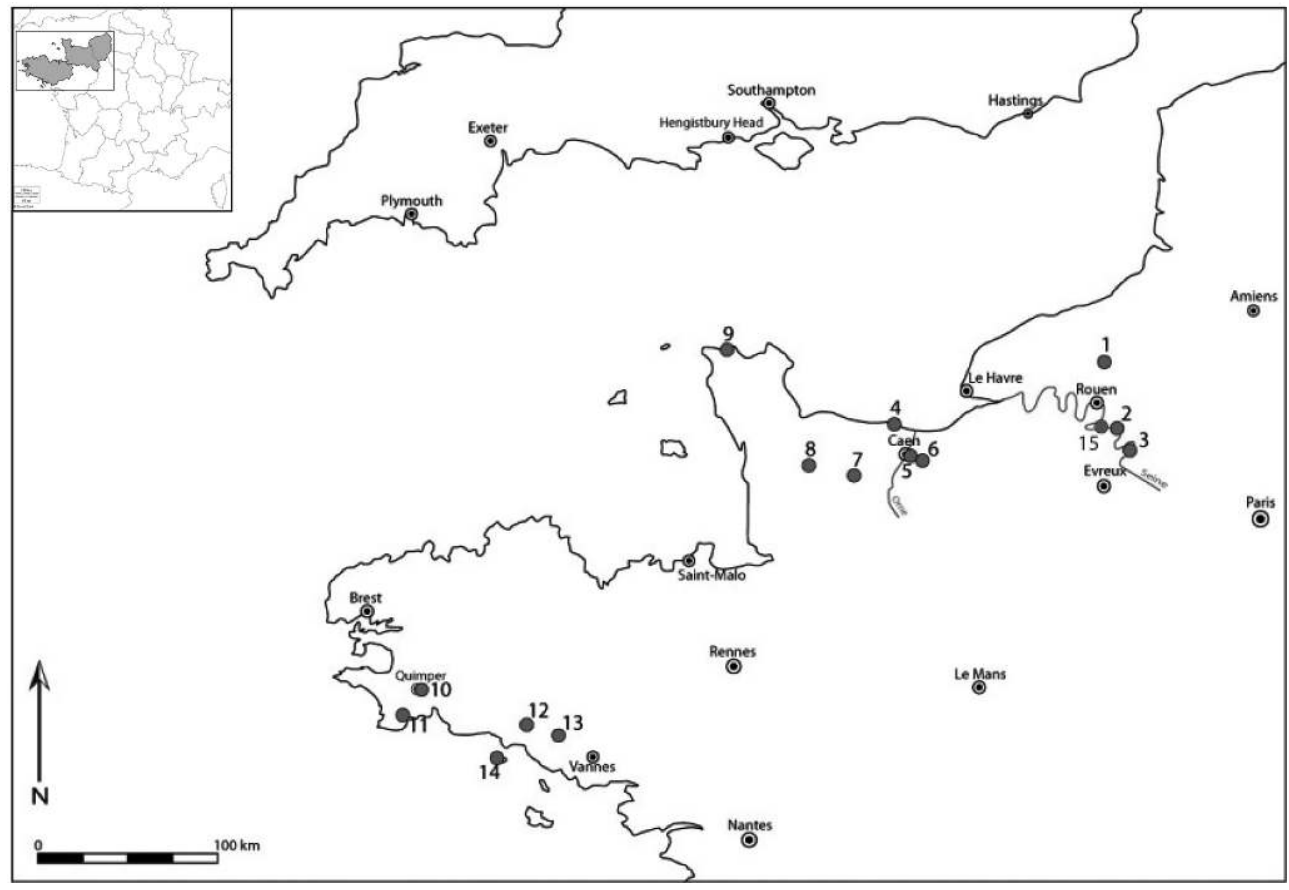

1 : Étaimpuis ; 2 : Bouafles ; 3 : Poses ; 4 : Courseulles-sur-Mer ; 5 : Ifs ; 6 : Cagny ; 7 : Cahagnes ; 8 : Agneaux ; 9 : Urville-Nacqueville ; 10 : Quimper ; 11 : Pont-l'Abbé ; 12 : Hennebont ; 13 : Pluvigner ; 14 : Kervédan; 15 : Alizay.

1: Étaimpuis; 2: Bouafles; 3: Poses; 4: Courseulles-sur-Mer; 5: Ifs; 6: Cagny; 7: Cahagnes; 8: Agneaux; 9: Urville-Nacqueville; 10: Quimper; 11: Pont-l'Abbé; 12: Hennebont; 13: Pluvigner; 14: Kervédan; 15: Alizay. D'après Godard, 2010.

La Haute-Normandie (fig. 5) a livré pour sa part une construction circulaire sur les sites d'Étaimpuis «La Briqueterie » (Blancquaert, 1995; Dechezleprêtre et al., 1997b) et de Bouafles « Les Mousseaux » (Fournier, 1996 ; Dechezleprêtre et al., 1997b). À Poses, au lieu-dit «Sur la Mare », cinq habitations circulaires ont été mises au jour (Bostyn, 1996; Dechezleprêtre et al., 1997b) tandis que sur le site d'Alizay, au moins deux maisons rondes sur poteaux plantés ont été identifiées ${ }^{3}$. 
Figure 5 : Plan des habitations circulaires de Haute-Normandie. Figure 5 : Roundhouses of Upper Normandy.

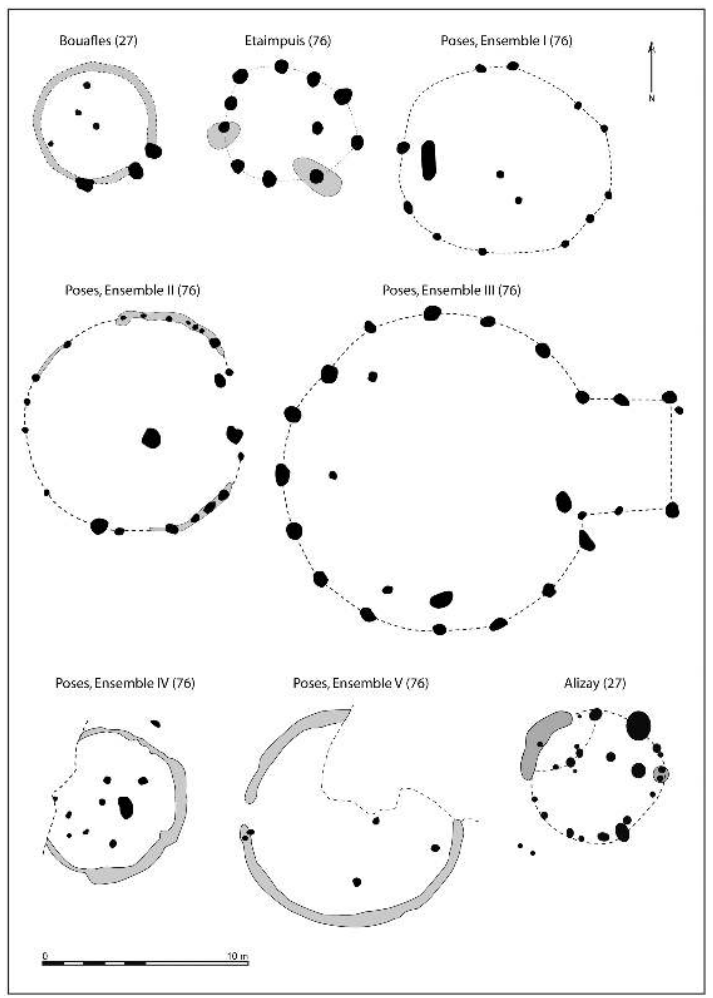

D'après Dechezleprêtre et al., 1997b ; V. Thieron in Marcigny, à paraître.

Des habitations circulaires ont également été découvertes en Basse-Normandie (fig. 6), comme à Agneaux, sur les lieux-dits «Bellevue » et « la Croix carrée » (Marcigny, 2000) et «la Tremblaye» (Ghesquière, 2001) qui comportent trois maisons rondes. À Cahagnes (Jahier, 1996; Lepaumier et al., 2002), c'est un véritable village d'au moins vingt-et-une habitations circulaires (fig. 7) qui a été mis au jour. Sur le site de «La Batterie Basse " à Urville-Nacqueville, plusieurs constructions ont également été identifiées (Lefort, 2010). « La Fosse Touzé » à Courseulles-sur-Mer (Jahier et al., 1997) a révélé deux habitations rondes tandis que les sites d'Ifs (Jahier, 1999 ; Lepaumier et al., 2002) et Cagny (San Juan et al., 1996; Lepaumier et al., 2002) ont livré chacun une maison circulaire. 
Figure 6 : Plan des habitations circulaires de Basse-Normandie. Figure 6 : Roundhouses of Lower Normandy.

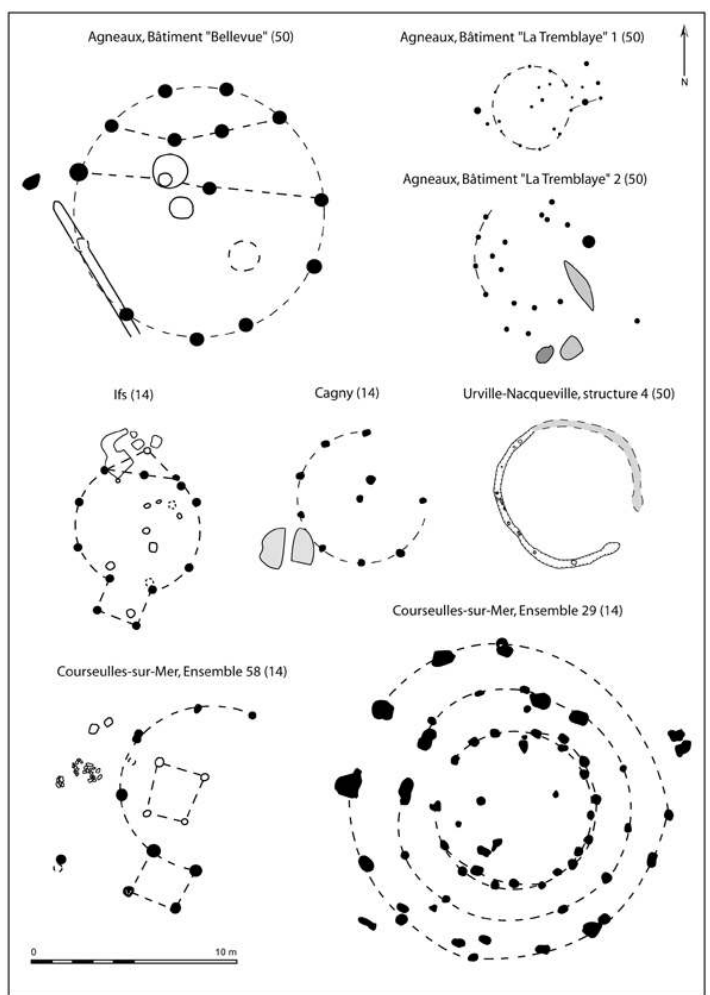

D’après J.-M. Richard in Marcigny et al., 2009 et Lepaumier et al., 2002, modifié ; Giazzon, 2004 modifié ; Jahier, 1997 modifié ; Lefort, 2010. 
Figure 7 : Plan général du site de Cahagnes (en haut) et illustration des différentes types de constructions circulaires sur poteaux présentes sur le site.

Figure 7 : General view of Cahagnes site (above) and illustration of the different kinds of roundhouses.

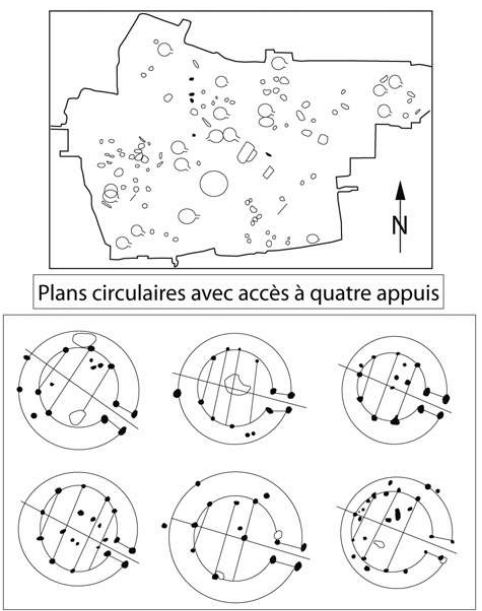

Plans circulaires avec accès à six appuis

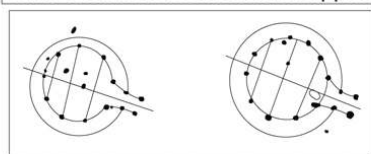

Plans Ovoïdes

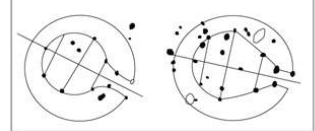

D’après Jahier et al., 1997 in Lepaumier et al., 2002.

Enfin, la Bretagne a également livré quelques structures que l'on pourrait interpréter comme des possibles maisons circulaires (fig. 8), comme sur le site de «Keralio » à Pont-l'Abbé (Hinguant et Le Goff, 1998), «Le Polvern » à Hennebont (Menez, 1987 ; Hinguant et Le Goff, 1998) et «Le Braden » à Quimper (Le Bihan, 1986), bien que la maison circulaire de ces deux derniers puissent également résulter de la superposition de deux bâtiments quadrangulaires. Les sites du " Talhouët» à Pluvigner (Tanguy, 1988) et de Kervédan ${ }^{4}$ sur l'île de Groix (Threipland, 1943) ont quant à eux livré une construction ronde en pierre. 
Figure 8 : Plan des habitations circulaires de Bretagne. Figure 8 : Roundhouses of Brittany.

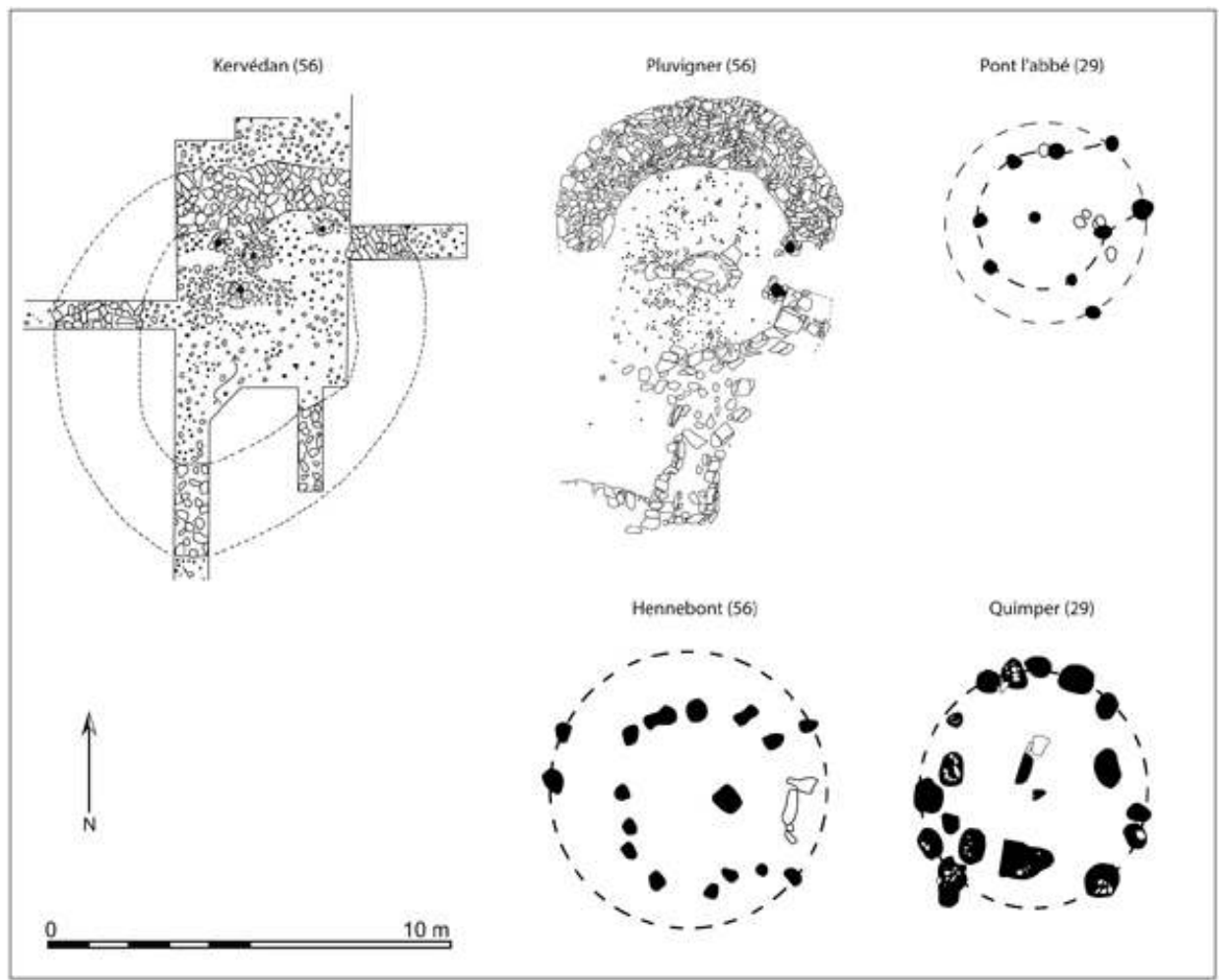

D’après Hinguant et al., 1998, modifié ; Threipland, 1943 ; Tanguy, 1988 ; Le Bihan, 1986, modifié.

\section{Première synthèse du corpus}

De ce corpus réactualisé des maisons circulaires, il se dégage avant tout une diversité des matériaux et des techniques de construction. Cette diversité s'inscrit à la fois au niveau des matériaux utilisés dans la structure (bois ou pierre, cette dernière n'ayant été notée qu'en Bretagne, ce qui est probablement lié aux ressources disponibles) et dans les techniques de construction (sur un ou plusieurs cercles de poteaux plantés, sur tranchée de fondation ou soubassements en pierre). À cela, il faut ajouter les observations effectuées sur l'aménagement interne de l'habitation, qui s'illustre pour certaines par des vestiges de partitions internes, par la présence ou non d'un poteau central ou encore par le nivellement du sol.

On remarquera ainsi, en plus de la présence d'un porche sur certains sites, une orientation majoritairement dirigée vers l'est et le sud-est (fig. 9) dans l'optimum de lumière et de confort mentionné par R. Pope pour l'Angleterre (Pope, 2007). On trouve également dans nos régions des habitations tournées vers l'ouest, direction généralement peu privilégiée car sujette aux vents dominants; dans la plupart des cas toutefois, ces constructions sont dotées d'un porche ou d'un auvent pour s'en protéger. C'est également leur dimension qui les rassemble et les standardise (fig. 10). En effet, parallèlement aux constructions recensées par R.Pope en Grande-Bretagne, les habitations de Bretagne et de Normandie mesurent, dans la majorité des cas, entre 7,5 et 10 mètres de diamètre, suivis de près par des structures plus petites allant de 5 à 7,4 mètres de diamètre. Ces dernières se trouvent souvent isolées sur le site, alors que 
les maisons de plus grande envergure (de 7,5 à 10 mètres) s'inscrivent dans un ensemble de plusieurs constructions, formant un petit hameau : faut-il alors voir, pour les premières, une implantation à plus court terme d'un petit groupe de personnes?

Figure 9 : Orientation des habitations circulaires de Bretagne et Normandie.

Figure 9 : Roundhouse orientations in Brittany and Normandy.

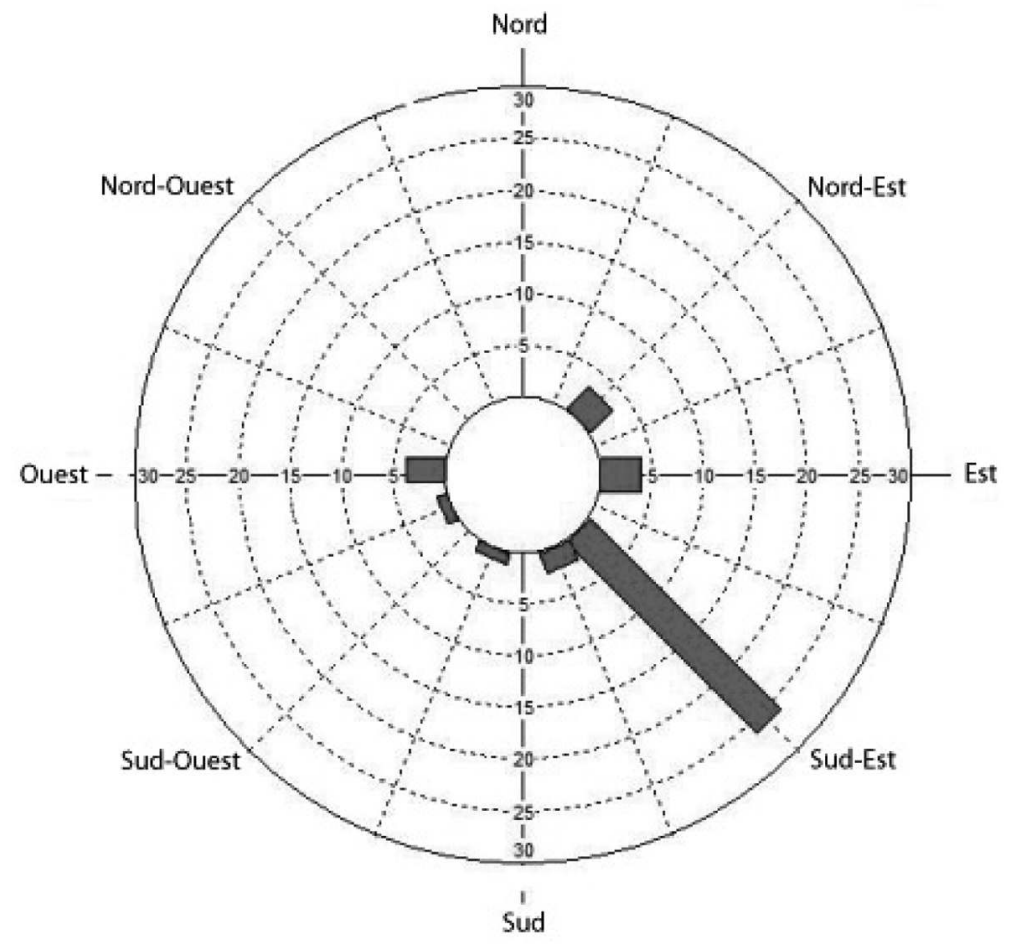

D'après Godard, 2010 
Figure 10 : Diamètres des constructions du corpus. Figure 10 : The diameters of the buildings in the author's corpus.

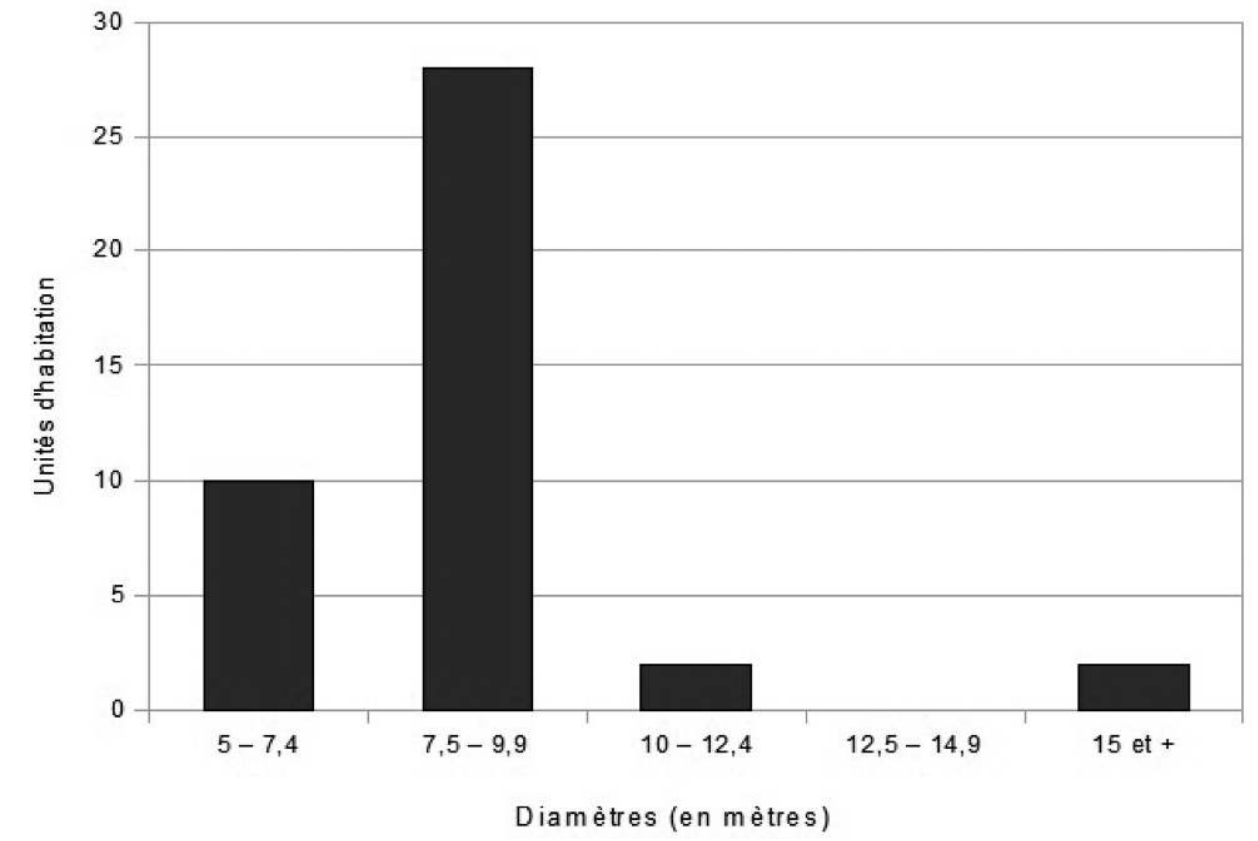

D'après Godard, 2010. comme à Poses, Cahagnes et Courseulles, sont rares et toujours insérées dans un ensemble, y jouant une place que l'on pourrait juger comme ostentatoire. Leur interprétation est malaisée, qu'il s'agisse d'un édifice à vocation communautaire ou d'une grande maison familiale, mais leur caractère domestique est avéré au vu du matériel retrouvé.

Enfin, nous remarquerons que nombre de ces sites, non contents de s'implanter dans des milieux favorables à la culture et l'élevage, se trouvent également à des points stratégiques de routes commerciales, notamment fluviales : ils se basent sur la côte, les îles, aux embouchures des fleuves ou le long de leur tracé. Toutes ces données réunies peuvent ainsi apporter de nouveaux éclaircissements sur l'origine de ces constructions et permettent d'observer leur rôle dans les échanges trans-Manche.

\section{L'habitat circulaire dans l'Ouest de la France : entre échange et culture}

\section{Des similarités typologiques avec la Grande-Bretagne}

L'inventaire préalablement établi rappelle que les constructions bretonnes et normandes, si elles présentent des profils extrêmement variés, interpellent moins par leur originalité que par leur ressemblance avec les constructions britanniques (fig. 11). Ainsi, l'habitation de Courseulles-sur-Mer, qui se base sur trois couronnes de poteaux plantés et possède un porche rentrant, rappelle les constructions sur trois cercles de poteaux de West Brandon, dans le comté de Durham (Cunliffe, 2005). De même, cette organisation multi-circulaire se retrouve dans l'habitation de Pimperne Down (Dorset) 
qui s'articule sur deux couronnes de poteaux (dont une troisième probable) et un porche court (Harding, 2009).

Figure 11 : Comparaison typologique des constructions de Courseulles-sur-Mer (Basse-Normandie) et de Pimperne Down en Angleterre.

Figure 11 : Typological comparison between the structures at Courseulles-sur-Mer (Lower Normandy) and Pimperne Down in England.

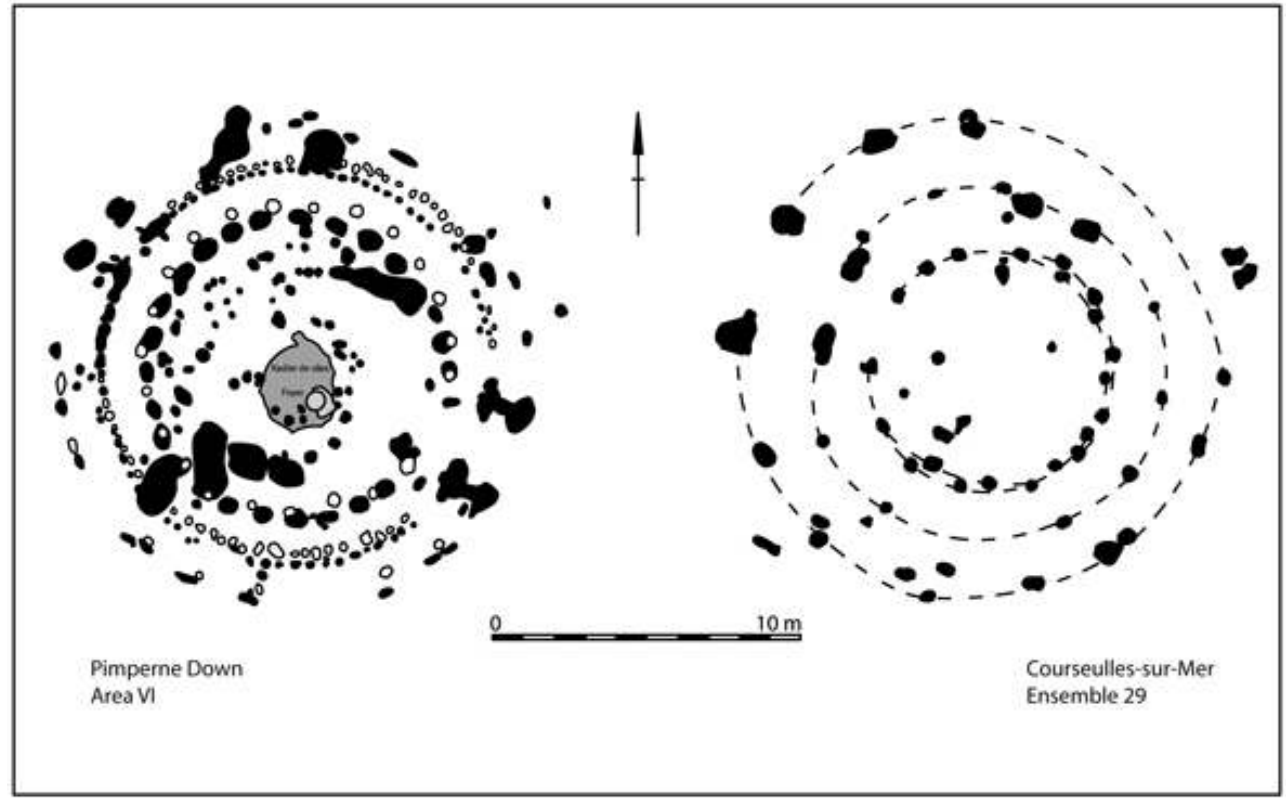

D’après Jahier et al., 1997 et Harding, 1993 standardisée, rappelant fortement (fig: 12) (Denbighshire). Ce site se caractérise par des constructions en série sur poteaux mesurant environ 6 à 10 mètres de diamètre (Guilbert, 1981) et dotées de porche sur deux ou quatre poteaux orientés au sud-est.

Des structures fossoyées comme à Bouafles (Seine-Maritime) ou à Urville-Nacqueville (Manche) ont également été découvertes dans le Sud de l'Angleterre, même si l'on retrouve plus fréquemment ces dernières dans le Nord de l'Angleterre et l'Écosse. Ainsi, le site de Scotstarvit (Fife) présente une habitation composée de trois couronnes, la première formée de trous de poteaux, les suivantes sur tranchées, formant ainsi une structure d'environ 18 mètres de diamètre (Harding, 2009). L'entrée, comme sur les deux sites normands, est matérialisée par un élargissement du fossé à chacune de ses extrémités, dans lesquelles devaient prendre place des paires de poteaux. Toutefois, la dimension monumentale de la maison de Scotstarvit contraste avec la taille modeste de nos constructions. Le site de West Plean (Stirlingshire) offre quant à lui une structure plus comparable en taille avec nos habitations ( 10 mètres de diamètre) mais celle-ci est composée d'un double cercle, sur tranchée et poteau (Cunliffe, 2005). Mais c'est peutêtre la taille modeste des constructions de Bouafles et de Nacqueville qui explique cette unique couronne; dans une maison d'une telle envergure, le double cercle n'est pas essentiel. L'exemple des constructions de Danebury (Hants) pourrait également apporter une autre explication, en particulier avec la maison CS1 où B. Cunliffe restitue une stake-wall house, qui n'est alors basée que sur une simple tranchée (Cunliffe, 2005). 
Figure 12 : Comparaison typologique des constructions de Cahagnes (Bronze final/Hallstatt) en Basse-Normandie et des habitations de Moel-y-Gaer (Hallstatt) au pays de Galles.

Figure 12 : Typological comparison between the structures at Cahagnes (Bronze final/Hallstatt) in Lower Normandy and the Early Iron Age houses of Moel-y-Gaer in Wales.

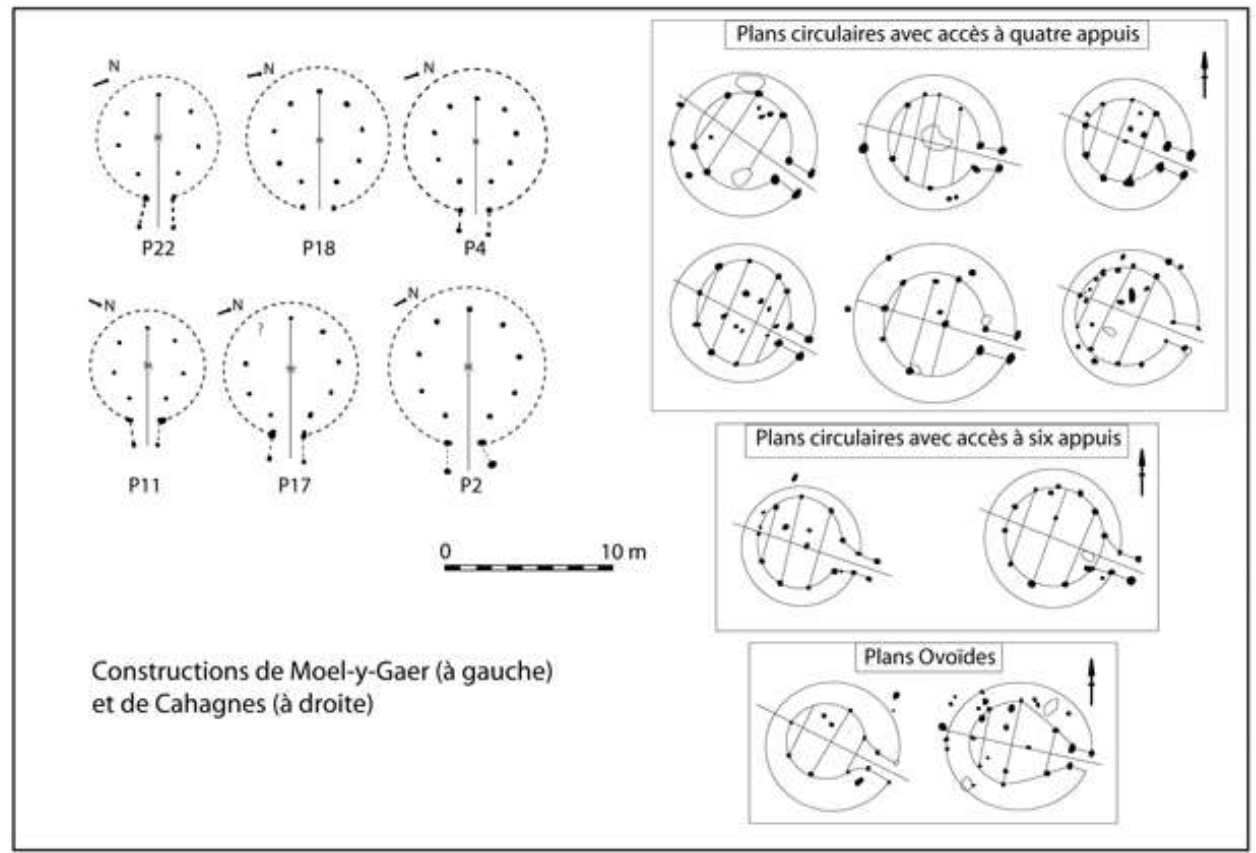

D’après Guilbert, 1982 et Jahier et al., 1997.

Enfin, en comparaison de nos deux structures en pierre, situées à Kervédan et à Pluvigner, nous pouvons observer quelques structures similaires (fig. 13) à Bodrifty (Cornouailles) ou à Kestor (Devon) où la pierre est utilisée pour les soubassements (Guilbert, 1981 ; Pope, 2008). Ces maisons circulaires sont basées sur un unique cercle de pierre, possèdent la même organisation interne (sans encellulement) que les maisons rondes de Bretagne et mesurent entre 6 et 10 mètres de diamètre (Cunliffe, 2005). 
Figure 13 : Comparaison typologique des structures circulaires de Bretagne et de Grande-Bretagne. Figure 13 : Typological comparison of round structures in Brittany and Great-Britain.

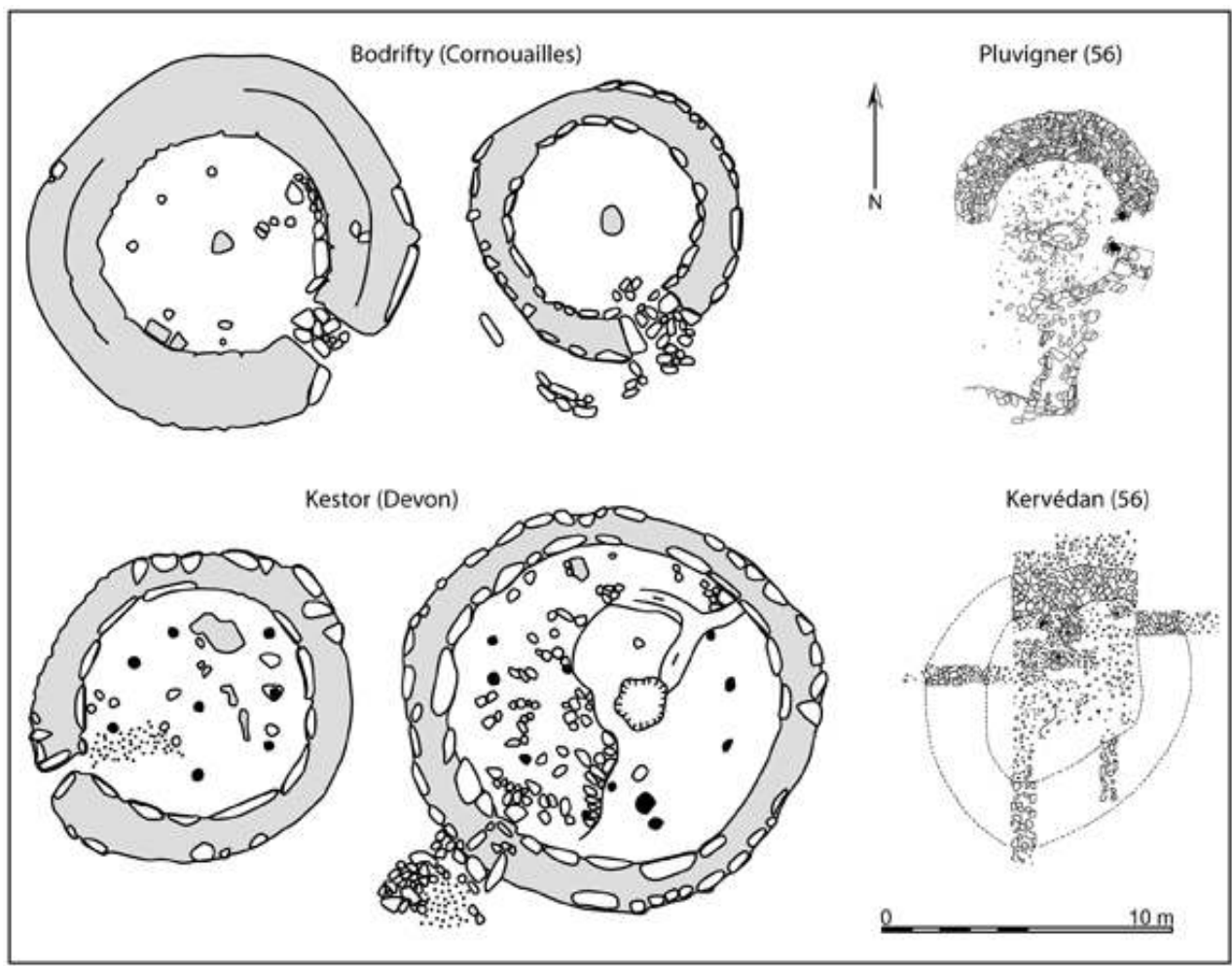

D’après Threipland, 1943 ; Tanguy, 1988, et d'après Cunliffe, 2005.

Toutefois, ces analogies ne sont pas suffisantes pour démontrer un réel lien culturel et social entre le continent et la Grande-Bretagne, car il faut également prendre en compte les périodes et les zones d'implantation des habitats circulaires.

\section{Comprendre le processus d'implantation}

Notre corpus, bien que le plus exhaustif possible, ne reflète pas, dans son nombre, la réalité de l'implantation des constructions. Mais ce hiatus n'est pas géographique ; il est chronologique. En effet, nos quinze sites, et leurs habitations, n'ont pas tous fonctionné en même temps. En replaçant chacune de ces structures dans l'époque qui leur est dévolue (fig. 14), il est alors possible d'observer s'il s'agit d'une mise en place progressive des habitations, au niveau géographique ou temporel, ou si l'on assiste au contraire à des vagues d'implantation correspondant à des périodes chronologiques précises. 
Figure 14 : Cartes de répartition des maisons circulaires en Bretagne et Normandie par périodes chronologiques.

Figure 14 : Location map of roundhouses in Brittany and Normandy by chronological period.
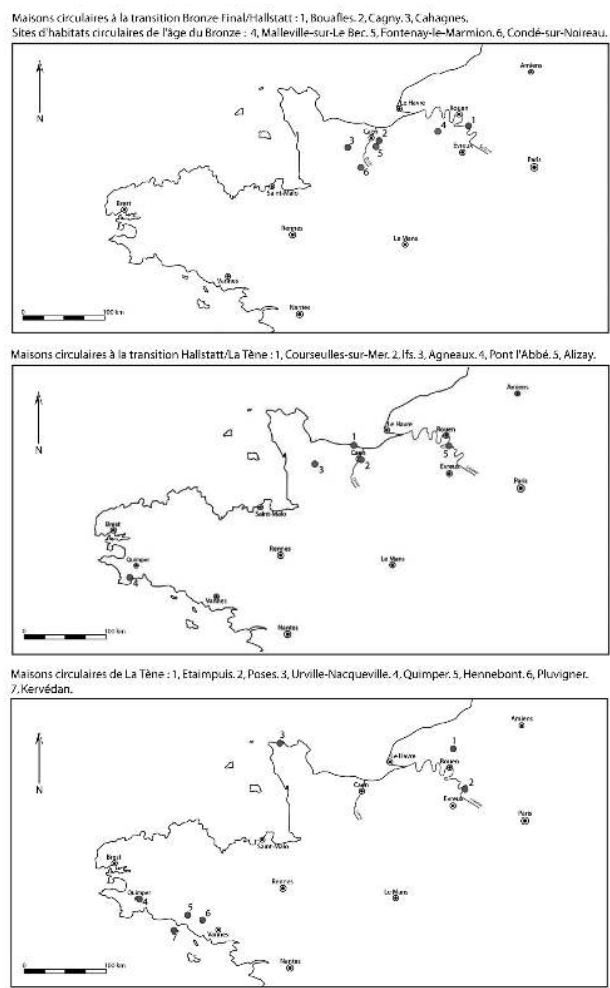

D’après Godard, 2010.

Hallstatt ancien, correspondent aux sites de Cahagnes et de Cagny (Basse-Normandie) ainsi que de Bouafles (Haute-Normandie). Des deux premiers sites, nous pouvons d'ores et déjà remarquer l'extrême proximité. Or, ces derniers s'implantent dans une région où l'on note déjà la présence de gisements circulaires d'envergure à l'âge du Bronze, à l'exemple des sites de Fontenay-le-Marmion ou Condé-sur-Noireau (Lepaumier et al., 2002). Les constructions de Cagny et de Cahagnes semblent donc s'inscrire dans la continuité des gisements circulaires déjà présents dans ce secteur. Le site de Bouafles semble quant à lui relativement isolé, même si l'on note la présence d'un gisement circulaire conséquent datant de l'âge du Bronze à Malleville-sur-le-Bec (à une quarantaine de kilomètres en aval).

Le premier âge du Fer semble relativement vide pour nos régions, que ce soit en Bretagne ou en Normandie. Toutefois, à la transition Hallstatt/La Tène (aux environs de 500-400 av. J.-C.), plusieurs sites se mettent en place, notamment Courseulles-sur-Mer (Basse-Normandie) suivi d'Agneaux et d'Ifs, toujours dans le même secteur. Malgré le peu de structures repérées pour le premier âge du Fer, c'est toujours la plaine de Caen qui semble privilégiée pour ces implantations bas-normandes. En Bretagne, le site de « Kéralio » à Pont-l'Abbé, présentant une possible maison circulaire, apparait dans un environnement vierge de ce type de constructions. La Haute-Normandie ne compte quant à elle que le site d'Alizay (Marcigny, à paraître).

48 
l'Abbé. Toutefois, à La Tène finale (vers 140 av. J.-C.), plusieurs structures circulaires telles que celles de Polvern, de Kervédan et de Quimper apparaissent, tandis qu'en Basse-Normandie, la tradition architecturale circulaire semble s'essouffler (elle n'est plus incarnée que par le site d'Urville-Nacqueville). Enfin, on notera la présence en Haute-Normandie des sites de Poses et d'Étaimpuis, deux implantations isolées.

À la lumière de ces répartitions chronologiques, nous remarquons que les constructions de la plaine de Caen semblent s'inscrire dans la continuité des structures circulaires de l'âge du Bronze dans la région; l'implantation dans ce secteur restera forte jusqu'à La Tène ancienne. À La Tène moyenne et finale, plusieurs constructions circulaires semblent s'établir sur la côte et les îles du sud de la Bretagne. La Haute-Normandie, elle, peine à faire surgir un schéma d'implantation, même si les sites de Poses et d'Étaimpuis se mettent en place dans le secteur au second âge du Fer.

Nous pouvons alors tenter de lier l'implantation des maisons circulaires, qui semble progressive au cours du Hallstatt et encore bien présente à la fin de La Tène, avec les ports et les zones d'échange commercial de l'âge du Fer.

\section{La maison ronde dans la dynamique des échanges trans-Manche}

51 La dynamique des échanges trans-Manche s'établit dès l'âge du Bronze au profit de la Grande-Bretagne (et tout particulièrement de la région des Cornouailles, au sud-ouest de l'Angleterre), mais également de la Bretagne et de la péninsule ibérique, qui regorgent d'étain et de cuivre. Un impressionnant couloir maritime, reliant toute la côte atlantique, se met alors en place, de l'Irlande au nord de l'Espagne (Cunliffe, 2007). En Angleterre, deux sites, Langdon Bay à Douvres (Kent) et Moor Sand à Salcombe (Devon) illustrent ces activités d'échange à l'âge du Bronze, matérialisées par des épaves contenant, pour le premier site, des artefacts en bronze et pour le second des barres de cuivre et d'étain (Muckelroy, 1981).

52 Nous n'aborderons pas ici les détails du commerce trans-Manche entre les îles Britanniques et le continent à l'âge du Fer, amplement étudié par plusieurs auteurs à l'image de B. Cunliffe (2007, 2009), K. Muckelroy (1981), C. Haselgrove (Haselgrove et Moore, 2007) ou encore J.C. Henderson (2007) et A. Lefort (2007). Notre intérêt se portera en particulier sur les points de contacts et d'échanges établis par les populations continentales et insulaires.

Pour le nord-ouest de la France, la Seine joue un rôle majeur, et ce, dès l'âge du Bronze. En effet, on notera la présence sur le site de Cannes-Écluses (Yonne), situé en amont de la Seine, de maisons circulaires datées de l'âge du Bronze (Marcigny et al., 2005) mais aussi de vestiges de commerce (avec des haches de l'est de la France, un poignard armoricain, etc.) illustrant des contacts à la fois avec l'ouest et l'est du continent. De même, les haches à talon, produit extrêmement développé à l'âge du Bronze en Normandie, se retrouvent tout le long de la Seine (Marcigny et al., 2005) et ont pu jouer un rôle important dans les échanges. Enfin, des exemples exceptionnels, tels les lunules en or originaires d'Irlande et datant de l'âge du Bronze, ont été retrouvés en Normandie, illustrant les contacts établis entre ces deux territoires (Verron, 2000). Enfin, l'embouchure de la Seine (près du Havre) semble avoir servi de plateforme de redistribution des artefacts vers les îles Britanniques et ce, jusqu'à l'âge du Fer si l'on s'en réfère à Strabon qui affirme que les marchandises sont transportées par la Seine 
jusqu'à la mer, d'où elles sont ensuite acheminées en Grande-Bretagne (Geographie, IV, $1,14)$.

Or, au premier âge du Fer, c'est le long de la Seine que s'établit le site de Bouafles, à seulement une centaine de mètres du fleuve, puis que se mettent en place à La Tène finale les sites de Poses et d'Étaimpuis. Même si ce dernier semble légèrement à l'écart de la Seine (environ vingt-cinq kilomètres), les deux premiers sites sont idéalement placés dans les méandres du fleuve, et ont probablement été les témoins et peut-être même les acteurs d'échanges commerciaux importants.

Pour la Basse-Normandie, les deux cours d'eau principaux qui retiennent notre attention sont l'Orne et la Sienne. En effet, à la transition de l'âge du Bronze et de l'âge du Fer, l'habitat de Cagny s'implante à quelques kilomètres de l'Orne, qui passe à Caen et se jette dans la Manche à Ouistreham (Calvados). La présence de lignite sur le site nous amène à suggérer des relations de commerce, peut-être avec les îles Britanniques, comme ce sera le cas pour le lignite d'Urville-Nacqueville plusieurs siècles plus tard. À la même époque que Cagny, le site de Cahagnes s'implante lui aussi dans la région, et sa céramique est extrêmement similaire à celle du premier gisement. Tout au long de l'âge du Fer, cette région reste fortement connectée à la dynamique des relations commerciales, peut-être par la présence au milieu de l'âge du Fer du site de Courseulles-sur-Mer, idéalement placé sur la côte, dont nous supposons que sa situation a dû lui permettre de nouer des contacts et de prendre part au réseau d'échanges, peut-être en lien avec les sites d'Agneaux et d'Ifs, si l'on en juge la céramique retrouvée sur le premier site. En effet, à Courseulles, neuf types céramiques sont à rapprocher de par leur morphologie à des séries britanniques (Jahier, 1997).

Toutefois, après plusieurs siècles de commerce intensif, le premier âge du Fer voit ces échanges diminuer, la demande en cuivre et en étain déclinant suite à une meilleure disponibilité des gisements de fer. Et si le négoce trans-Manche perdure, comme l'illustrent par exemple les "Currency Bar » (lingots de fer), retrouvés à Cormelles-leRoyal (près de Caen) ou à Bretteville-sur-Odon, il est toutefois beaucoup moins soutenu (Cunliffe, 2005).

57 Le commerce trans-Manche va reprendre de façon importante au second âge du Fer (fig. 15), avec la mise en place de nouveaux sites portuaires en Angleterre, comme Mount Batten (Devon), Portland (Dorset), Poole Harbour (Dorset) et bien sûr Hengistbury Head (Dorset). Ce dernier site portuaire va trouver son pendant continental à Alet et à Urville-Nacqueville, où les mêmes types d'artefacts qu'à Hengistbury Head ont été retrouvés: des amphores de type Dressel 1A, un statère vénète et des bracelets de lignite issus des falaises littorales de Kimmeridge (Dorset) furent retrouvées à Nacqueville (Lefort, 2010), tandis que des céramiques à cordon ont été découvertes à Alet, dans l'estuaire de la Manche. 
Figure 15 : Carte de répartition des sites portuaires impliqués dans le commerce trans-Manche à la fin de La Tène ainsi que des sites comportant des maisons circulaires à la même période.

Figure 15 : Location map of harbours implicated in cross-Channel trade at the end of La Tène period and contemporary roundhouse sites.

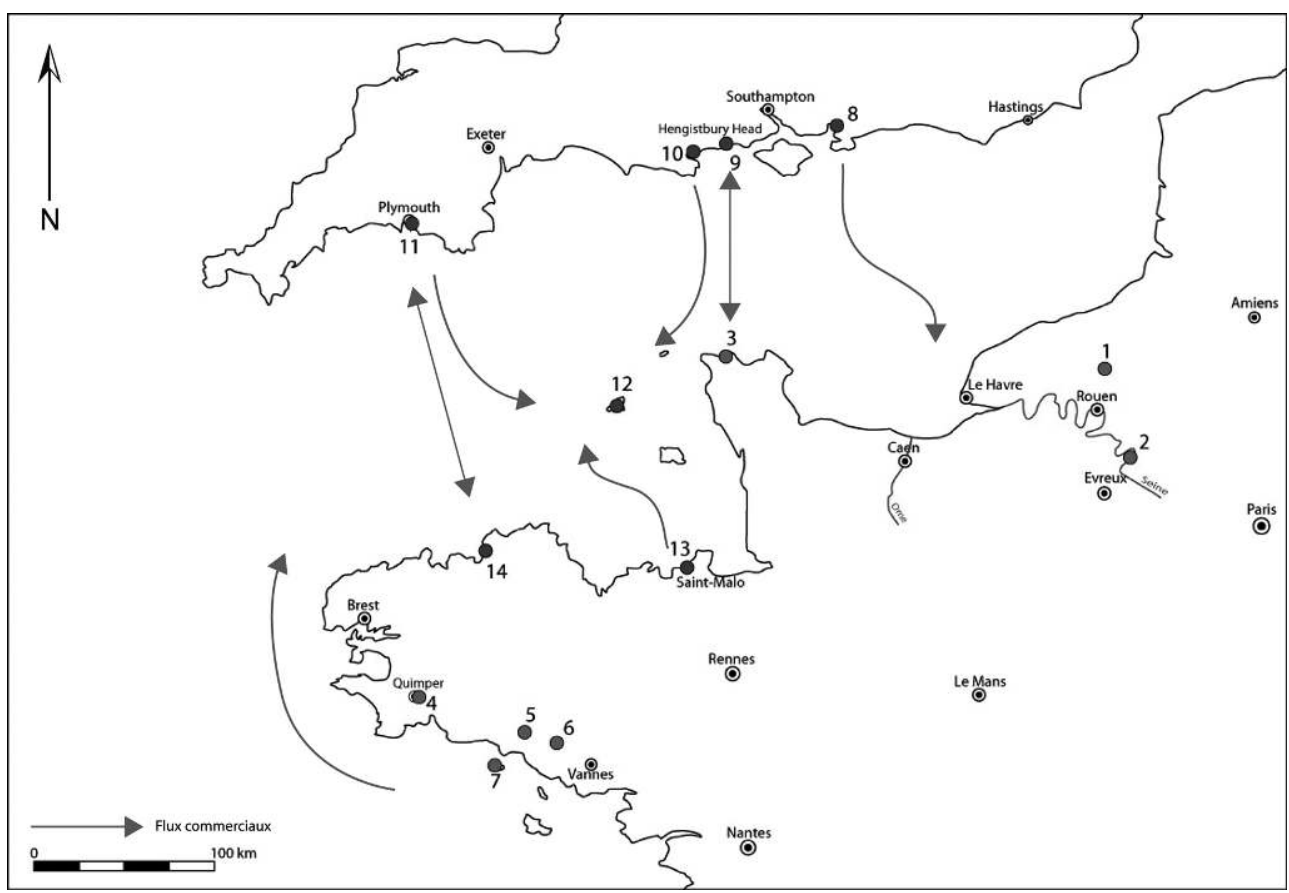

Les maisons circulaires : 1 : Étaimpuis ; 2 : Poses ; 3 : Urville-Nacqueville ; 4 : Quimper ; 5 : Hennebont ; 6 : Pluvigner ; 7 : Kervédan. Les sites portuaires : 8 : Chichester ; 9 : Hengistbury Head ; 10 : Poole Harbour ; 11 : Mount Batten ; 12 : Guernsey ; 13 : Alet ; 14 : Le Yaudet.

Roundhouses: 1: Étaimpuis; 2: Poses; 3: Urville-Nacqueville; 4: Quimper; 5: Hennebont; 6: Pluvigner; 7:

Kervédan. Harbours: 8: Chichester; 9: Hengistbury Head; 10: Poole Harbour; 11: Mount Batten; 12: Guernsey; 13: Alet; 14: Le Yaudet.

D'après Godard 2010 ; Cunliffe, 1993 et Lefort, 2007.

Concernant la Bretagne, B. Cunliffe fait état de la répartition des amphores Dressel 1 sur la côte sud (Cunliffe, 1993). Il apparaît que ces dernières sont particulièrement concentrées en trois points, et plus particulièrement sur la presqu'île de Quiberon.

Or, c'est dans cette région appartenant au territoire des Vénètes que s'établissent à La Tène les habitats circulaires de Kervédan, Hennebont et Pluvigner, possédant chacun un accès à la mer et sur lesquels on a retrouvé des céramiques similaires à celles observées au Petit-Celland (pour Kervédan) ou au profil à rapprocher avec les céramiques britanniques (comme à Pluvigner). Parallèlement, sur le territoire des Osismiens, le site du «Braden " à Quimper fait état d'une maison circulaire et il en est de même pour Pont-l'Abbé, situé au bord de la mer à une vingtaine de kilomètres. À Pont-l'Abbé, les archéologues ont d'ailleurs retrouvé les vestiges de bracelets en lignite ainsi que de haches à douilles armoricaines (Hinguant et Le Goff, 1998).

Ainsi, c'est un lien commercial fort qui peut être observé entre la Normandie, la Bretagne et la Grande-Bretagne, relation privilégiée qui se matérialise par des importations et des exportations de céramiques, des artefacts en bronze ou encore des pièces de monnaies pour les périodes les plus récentes. Nous noterons que les maisons circulaires sont implantées pour la majeure partie dans des zones de fort contact économique avec les îles Britanniques, ce qui nous amène à une ultime réflexion sur leur origine. 


\section{Un héritage britannique, une culture commune atlantique ou une tradition indigène?}

61 Plusieurs arguments font pencher la balance en faveur d'une culture commune des deux côtés de la Manche, ce qui expliquerait que l'on retrouve des habitations circulaires sur le sol français semblables à celles des îles Britanniques. Ainsi, une culture uniforme (la "culture atlantique ») a été identifiée voilà plus d'une dizaine d'années (Giot et al., 1995), unissant la façade atlantique, parallèlement à une culture allant de l'Alsace au Bassin parisien nommée RSFO (Rhin-Suisse-France orientale) s'établissant dès l'âge du Bronze.

Dès le Campaniforme, de nombreux liens s'établissent entre le continent et la GrandeBretagne. Ces affinités s'illustrent notamment par le phénomène des tombes princières (Marcigny et Talon, 2009) que l'on retrouve à la fois dans la région du Wessex (Angleterre) et en Bretagne, mais également aux Pays-Bas et en Belgique. Ces similitudes s'incarnent également dans les maisons rondes, qui apparaissent sur la façade atlantique. Ces dernières, qui s'établissent en Grande-Bretagne à la fin du Néolithique, se retrouvent à la même époque dans la péninsule ibérique et sur nos côtes au moins au Bronze ancien. Les maisons espagnoles, de petite envergure (environ 3 à 4 mètres de diamètre) connaîtront d'ailleurs le même phénomène de monumentalisation (Ayan, 2006) qui touchera la Grande-Bretagne et l'Ouest de la France à la transition de l'âge du Bronze et du Fer.

63 Mais le nord de la culture atlantique à l'âge du Bronze ancien et final présente tant de similarités que C. Marcigny a proposé d'y voir un complexe spécifique, « Manche - mer du Nord» («MMN»), qui rassemblerait les îles Britanniques, le nord-ouest du continent ainsi que le Benelux (Marcigny et Talon, 2009). Ce complexe rassemble également le groupe "Deverel-Rimbury", qui se caractérise par des structures funéraires, des céramiques (urnes et seaux globulaires, à cordons par exemple) et des structures d'habitats, avec des implantations rurales encloses, disposant notamment d'un habitat circulaire et s'entourant d'annexes et de parcellaires.

C'est donc une culture uniforme qui semble être en place à l'âge du Bronze pour ces régions, liées par des relations commerciales, sociales et culturelles. Même l'émergence, au Bronze final, d'une culture dans l'Est de la France (la culture «nordalpine ») ne semble pas perturber le Grand Ouest qui continue son évolution parallèle et similaire, à l'image des fortifications britanniques (les « ring-forts ») qui semblent se mettre en place également sur le territoire français, comme l'illustre le site de Malleville-sur-le-Bec (Eure).

65 Dès lors, le premier âge du Fer semble s'inscrire dans la continuité des complexes atlantiques de l'âge du Bronze. Quoi de plus naturel, donc, que les maisons rondes de l'âge du Fer soient issues de cette culture commune?

66 Toutefois, on assiste à une véritable rupture entre le premier âge du Fer et le second. Cette dernière se caractérise à la fois par la baisse des échanges trans-Manche mais aussi par une uniformité générale de la culture de La Tène aussi bien dans l'Ouest de la France que dans le reste du continent (Milcent, 2006). L'habitat circulaire, au milieu du premier âge du Fer, n'est plus qu'anecdotique sur le territoire, s'énumérant au comptegoutte, alors qu'en Espagne, de véritables villages, composés d'une vingtaine de maisons en pierre, se mettent en place et sont si significatifs qu'on les identifie à la 
" culture des castros ». Cette implantation de grande envergure, que l'on retrouve en Grande-Bretagne, est inexistante en Normandie et Bretagne (à l'exception de Cahagnes, au début de l'âge du Fer), alors que ces deux régions semblaient si liées quelques siècles plus tôt. Et si un temps une culture en "vase-clos » selon l'expression d'I. Jahier, a été envisagée (Jahier, 1997), il semble plutôt que l'on assiste à l'évolution indépendante d'une tradition issue d'une même culture, établie à l'âge du Bronze.

Quoi qu'il en soit, le second âge du Fer, dont nombre des habitations circulaires de notre corpus est issu, fait apparaître une implantation totalement différente de ces structures en comparaison avec celles de l'âge du Bronze et du premier âge du Fer. En effet, en Bretagne et en Normandie, elles se concentrent plutôt sur des zones côtières ou fluviales et à des points de contacts participant au commerce trans-Manche. Nous ne sommes plus ici face à une culture uniforme mais plutôt face à des contacts par la navigation et le cabotage.

Ainsi, nous pensons, comme le mentionne B. Cunliffe (2009), que la culture atlantique et le complexe MMN de l'âge du Bronze s'effacent à l'âge du Fer permettant aux différentes régions de se développer chacune de leur côté. La péninsule ibérique et la Grande-Bretagne, relativement isolées du monde continental, vont suivre une évolution globalement similaire tout en développant leurs propres particularités, notamment au niveau architectural, tandis que la culture atlantique va peu à peu décliner dans l'Ouest de la France (et en particulier en Bretagne et en Normandie) au profit d'une culture laténienne. Toutefois, ces régions ne se ferment pas au commerce, comme le montrent les échanges intensifs qui se mettent en place au second âge du Fer.

Enfin, le retour des constructions circulaires à la fin de La Tène, en faible nombre mais particulièrement notables sur les points de contact, pourrait illustrer l'installation à court ou moyen terme de populations britanniques opérant au niveau commercial et artisanal dans ces régions, ce qui expliquerait la présence d'habitations isolées et non pas de villages groupés. Ainsi amenées à s'implanter sur le continent, ces populations auraient bâti une structure qui leur est intrinsèquement familière: une maison circulaire.

\section{Conclusion}

70 L'implantation de ces maisons circulaires est à la fois affaire de culture et d'héritage architectural. En effet, les maisons circulaires de la fin de l'âge du Bronze et du début de l'âge du Fer, comme celles situées en Basse-Normandie, sont directement issues de la culture Manche - mer du Nord, tandis que les autres implantations sont fortement liées aux échanges commerciaux mis en place tout au long de l'âge du Fer, au moyen des sites portuaires et des lieux de contacts économiques (voies fluviales et maritimes). Ces sites de la fin de l'âge du Fer pourraient ainsi correspondre à des comptoirs, mis en place par des populations britanniques et assurant ainsi le lien entre le continent et les îles.

71 Tandis que l'archéologie poursuit son travail d'étude du monde rural, peut-être auronsnous la chance, dans les années à venir, de voir apparaître de nouvelles constructions circulaires qui éclaireront un peu plus nos connaissances sur ce type d'habitat à l'âge du Fer. En Grande-Bretagne, les recherches se poursuivent sur la thématique de la maison ronde et se concentrent désormais sur l'organisation de l'espace de vie et les rites liés à l'habitat (comme les dépôts de mobilier et les rites d'abandon) tout en 
reconsidérant les théories cosmologiques proposées dans les années 1980 (Pope, 2007 ; Webley, 2007).

\section{BIBLIOGRAPHIE}

AUDOUZE F. et BUCHSENSCHUTZ O., 1989 - Villes, villages et campagnes de l'Europe celtique, Hachette, Paris, 362 pages.

AyAN X. M., POPE R. et AlBERRO Y. M., 2006 - « Una Edad del Hierro redonda: la cabaña circular en los castros del NW de la Península ibérica », Kalathos 24-25, Revista del S.A.E.T, p. 177-217.

BERSU G., 1940 - « Excavations at Little Woodbury, Wiltshire », Proceedings of the Prehistoric Society, 6, p. 30-111.

BLANCQUAERT G., 1995 - Un secteur champêtre et les restes d'une forge à Étaimpuis « La Briqueterie » (Seine-Maritime), Document final de synthèse, Sauvetage urgent, Petit-Quevilly, SRA-DRAC HauteNormandie.

BRIARD J., NicolaRdot J.-P. et RoBino P., 1988 - « Habitats de l'âge du Bronze en Bretagne : « Le Vivier » (Quiberon) et « Grosse-Roche » (Saint-Jacut) », in BUCHSENSCHUTZ O. et AudouZE F. (dir.), Architecture des âges des métaux : fouilles récentes, Dossiers de protohistoire, 2, Errance, Paris, p. 9-18.

Collis J., 1990 - « L'impact des processus d'urbanisation sur les sites ruraux : le cas d'Owslebury, Hants, Angleterre », Revue archéologique de l'Ouest, Supplément n³, p. 209-222.

CUNLIFFE B., 1993 - La Gaule et ses voisins : le grand commerce dans l'Antiquité, traduction de F. Vidal, Collection Antiquité/Synthèse n ${ }^{\circ}$ 4, Picard, Paris, 253 pages.

CUNLIFFE B., 2005 - Iron Age communities in Britain. An account of England, Scotland, and Wales, from the seventh century $B C$ until the Roman conquest, $4^{\mathrm{e}}$ édition, Routledge, Londres, 741 pages.

CUNLIFFE B., 2007 - « Continent cut off by fog:just how insular is Britain? », Scottish Archaeological Journal, 29 (2), p. 99-112.

CUNLIFFE B., 2009 - « Looking forward: maritime contacts in the first millenium BC », in CLARK P. (dir.), Bronze Age Connections: Cultural Contact in Prehistoric Europe, Oxbow books, p. 80-93.

DeChezleprêtre T., Cousyn P. et LÉON G., PAEZ-REZENDE L. et ROUGIER R., 1997a - « Architecture des bâtiments de l'âge du Fer en Haute-Normandie ", in MARION S. et BLANCQUAERT G. (dir.), Les installations agricoles de l'âge du Fer en France septentrionale, actes du colloque de Paris, 2000, Paris, p. 321-338.

Dechezleprêtre T., Billard C., Blancquaert G., Fournier P. et LANGlois J.-Y. 1997b - « Les constructions à plan circulaire de Haute-Normandie », Revue archéologique de l'Ouest, 14, p. 49-56. DECHEZlEPRÊTRE T. et GINOUX N., 2005 - « Les constructions circulaires de la moitié nord de la France : état de la question », in BUCHSENSCHUTZ O. et MORDANT C. (dir.), Architectures protohistoriques en Europe occidentale du Néolithique final à l'âge du Fer, Actes des congrès 
internationaux des sociétés historiques et scientifiques, $127^{\mathrm{e}}$, Nancy 2002 , Comité des travaux historiques et scientifiques, p. 77-87.

DREWETT P.-L., 1990 - « La fouille d'un habitat du Bronze final à Black Patch, East Sussex, Angleterre ", in ROULIÈRE-LAMBERT M.-J. et OBERKAMPF M. (dir.), Un monde villageois. Habitat et milieu naturel en Europe de 2000 à 500 av. J.-C., Cercle Girardot, Lons-le-Saunier, p. 31-35.

Duval A., LE BIHAN J.-P. et MENEZ Y., 1988 - Les Gaulois d'Armorique, la fin de l'âge du Fer en Europe tempérée, Actes du XII colloque de l'A.F.E.A.F, Quimper, mai 1988.

GHESQUIÈRE E., 2001 - Fouille de sauvetage sur le site d'Agneaux « la Tremblaye », janvier-février 2001, Document final de synthèse 1354, Caen, SRA/DRAC Basse-Normandie.

Giazzon D., 2004 - Fouille sur le site d'Agneaux, «la Tremblaye », Février 2004, Document final de synthèse 1613, Caen, SRA/DRAC Basse-Normandie.

GIOT P.-R., BRIARD J. et PAPE L., 1995 - Protohistoire de la Bretagne, Ouest-France université, Rennes, 423 pages.

GODARD C., 2010 - L'habitat circulaire en Bretagne et Normandie à l'âge du Fer : un héritage britannique? Étude, inventaire et remise en contexte des constructions dans la dynamique des échanges trans-Manche, Mémoire de master 1, université de Haute-Bretagne, Rennes, 105 pages.

GUILBERT G.-C., 1981 - « Double ring roundhouses, probable and possible in Prehistoric Britain », Proceedings of the Prehistoric Society, 47, p. 299-387.

HARDING D.W., BLAKE I.M. et ReYNOLDS P.J., 1993 - An Iron Age settlement in Dorset. Excavation and Reconstruction, Department of Archaeology, University of Edinburgh, 120 pages.

HARDING D.W., 2009 - The Iron Age Round-house: Later Prehistoric Building in Britain and Beyond, Oxford University Press, 345 pages.

HASELGROVE C. et MOORE T., 2007 - The Later Iron Age in Britain and Beyond, Oxbow books, Oxford, 534 pages.

Henderson J.-C., 2007 - « The Atlantic West in the Early Iron Age », in HASElgRove C. et Pope R. (dir.), The Earlier Iron Age in Britain and the near continent, Oxbow books, p. 306-324.

HINGUANT S. et LE GOFF E., 1998 - « Un site de l'âge du Fer stratifié en milieu rural. L'établissement de Keralio à Pont-l'Abbé (Finistère) », Revue archéologique de l'Ouest, 15, p. 59-114.

HODARA J.-J., 2005 - « L'architecture de l'habitat protohistorique : contraintes techniques », in BUCHSENSCHUTZ O. et MORDANT C. (dir.), Architectures protohistoriques en Europe occidentale du Néolithique final à l'âge du Fer, Actes des Congrès nationaux des sociétés historiques et scientifiques, $127^{\mathrm{e}}$, Nancy, 2002, Comité des travaux historiques et scientifiques, p. 65-76. JAHIER Y., 1996 - Fouille de sauvetage sur le site de Cahagnes, Document final de synthèse, Caen, SRADRAC Basse-Normandie.

JAHIER Y., 1997 - Fouille de sauvetage sur le site de Courseulles-sur-Mer, « La fosse Touzé », Document final de synthèse 1198, Caen, SRA-DRAC Basse-Normandie.

Jahier Y., Besnard-Vauterin C.-C., Lepaumier H., Paris P., Renault V., SAn juan G., Dron J.-L., Hincker V. et FoRfAIT N., 1997 - « Les bâtiments de l'âge du Fer en Basse-Normandie : panorama des découvertes ", in MARION S. et BLANCQUAERT G. (dir.), Les installations agricoles de l'âge du Fer en France septentrionale, Actes du colloque de Paris, 2000, Paris, p. 339-357.

JAHIER Y., 1999 - Fouille de sauvetage à Ifs, ZAC Object'Ifs sud (Calvados), mars 1999, Document final de synthèse 1194, Caen, SRA-DRAC Basse-Normandie. 
LE BIHAN J.-P., 1986 - Fouille programmée au Braden, Rapport de fouilles, Rennes, SRA-DRAC de Bretagne.

LEFORT A., 2007 - Les relations trans-Manche à la fin de l'âge du Fer. Les relations entre la Gaule et le Sud de l'Angleterre et les rares éléments métalliques bretons retrouvés sur le continent : problèmes d'interprétation, Mémoire de master 1, université de Bourgogne, Dijon, 143 pages.

LEFORT A., 2010 - Opération de fouilles archéologiques sur l'estran d'Urville-Nacqueville, Rapport final d'opération, Caen, SRA-DRAC Basse-Normandie.

LEPAUMIER H., MARCIGNY C. et GHESQUIÈRE E., 2002 - « L'architecture des habitats protohistoriques de Normandie : quelques exemples de la fin du $\mathrm{III}^{\mathrm{e}}$ millénaire au début du second âge du Fer ", in BUCHSENSCHUTZ O. et MORDANT C. (dir.), Architectures protohistoriques en Europe occidentale du Néolithique final à l'âge du Fer, Actes des Congrès nationaux des sociétés historiques et scientifiques, $127^{\mathrm{e}}$, Nancy, 2002, Comité des travaux historiques et scientifiques, p. 231-264.

MARCIGNY C., 2000 - Fouille de sauvetage sur le site d'Agneaux «Bellevue et la Croix carrée » septembre 2000, Document final de synthèse 1311, Caen, SRA-DRAC Basse-Normandie.

MARCIGNY C. et GHESQUIÈRE E., 2003 - L'île Tatihou (Manche) à l'âge du Bronze - habitats et occupation du sol, Documents d'archéologie française, Maison des sciences de l'homme, 192 pages.

Marcigny C., Colonna C., Ghesquière E. et Verron G., 2005 - La Normandie à l'aube de l'histoire. Les découvertes archéologiques de l'âge du Bronze 2300-800 av. J.-C., Somogy, Paris, 151 pages.

MARCIGNY C. et TALON M., 2009 - «Sur les rives de la Manche. Qu'en est-il du passage de l'âge du Bronze à l'âge du Fer à partir des découvertes récentes? » in DAUBIGNEY A., MILCENT P.-Y., TALON M. et VITAL J. (dir.), De l'âge du Bronze à l'âge du Fer en France et en Europe occidentale ( $X^{e}$-VIII ${ }^{e}$ siècle av. J.C.). La moyenne vallée du Rhône aux âges du Fer, Actes du XXX ${ }^{e}$ colloque de l'A.F.E.A.F (Saint-Romainen-Gal, 26-28 mai 2006), p. 345-403.

Menez Y., Daire M.-Y, Hyvert J., LANgouet I., Le Bihan J.-P. et TANGuY D., 1990 - « Les bâtiments de l'âge du Fer en Armorique ", Revue archéologique de l'Ouest, Supplément n³ 3, p. 121-137.

MiLCENT P.-Y., 2006 - « Premier âge du Fer médio-atlantique et genèse multipolaire des cultures matérielles laténiennes ", in Vitali D. (dir.), Celtes et Gaulois, l'archéologie face à l'histoire, 2 : la préhistoire des Celtes, Actes de la table ronde de Bologne-Monterenzio, 28-29 mai 2005. Glux-enGlenne : Bibracte, Centre archéologique européen, p. 81-105.

Petit C., 2003 - « Molesme "Sur-les-Creux" des banquets gaulois aux poissons gallo-romains », Les dossiers de l'Archéologie, $\mathrm{n}^{\circ}$ 284, p. 90-97.

POPE R., 2007 - « Ritual and the roundhouse: a critique of recent ideas on the use of domestic space in Later British prehistory ", in HASELGROVE C. et POPE R. (dir.), The Earlier Iron Age in Britain and the near continent, Oxbow books, p. 204-228.

POPE R., 2008 - « Roundhouses, 3000 years of prehistoric design », Current Archaeology, 222, p. 14-21.

RALSTON I. et PoPe R., 2005 - « La pierre et le bois dans les maisons en Grande-Bretagne », in BUCHSENSCHUTZ O. et MORDANT C. (dir.), Architectures protohistoriques en Europe occidentale du Néolithique final à l'âge du Fer, Actes des congrès nationaux des sociétés historiques et scientifiques, $127^{\mathrm{e}}$, Nancy, 2002, Comité des travaux historiques et scientifiques, p. 41-48.

SAN JUAN G., GHESQUiÈRE E. et MÉNIEL P., 1996 - « Un site d'habitat protohistorique avec un cercle de trous de poteaux à Cagny (Calvados) ", Revue archéologique de l'Ouest, 13, p. 89-102. 
TANGUY D., 1988 - «L'habitat du second âge du Fer en Pluvigner (Morbihan) », Architectures des âges des Métaux : fouilles récentes, Dossiers de protohistoire, 2, Paris, p. 71-80.

THREIPLAND L.-M., 1943 - « Excavations in Brittany, spring 1939 », Archaeological Journal, 100, p. 128-149.

VERRON G., 2000 - Préhistoire de la Normandie, Ouest-France, Rennes, 364 pages.

WEBLEY L., 2007 - « Using and abandoning Roundhouses: a reinterpretation of the evidence from late Bronze Age - Early Iron Age southern England », Oxford Journal of Archaeology, 26 (2), Oxford, p. $127-144$

\section{NOTES}

1. Il est également possible que Strabon ait voulu évoquer la forme des toitures de ces constructions, mais rien n'est avéré.

2. Dans The first farms, [http://www.historic-cornwall.org.uk/flyingpast/first.html].

3. Marcigny C., Rapport final d'opération, Inrap, à paraître.

4. L'interprétation du bâtiment de Kervédan reste cependant sujette à discussion, car il pourrait également s'agir d'un bâtiment quadrangulaire aux angles arrondis.

\section{RÉSUMÉS}

La découverte de plus en plus fréquente de maisons circulaires sur le sol français et, en particulier, en Bretagne et en Normandie, est à l'origine de nombreuses interrogations sur la présence de ces habitations dans nos régions, alors que ces structures sont bien connues dans les îles Britanniques. À l'aide d'un inventaire des maisons circulaires de l'âge du Fer et d'une étude plus complète de leur architecture, notre volonté est ici d'identifier l'origine de ces constructions tout en appréhendant au mieux leur place au sein des relations trans-Manche.

Il apparaît alors, après comparaison typologique, que ces constructions présentent de nombreuses similitudes avec les structures de Grande-Bretagne. Leur emplacement, le long des fleuves et de la côte, ainsi que la découverte d'artefacts d'importations britanniques viennent appuyer l'hypothèse d'une tradition architecturale issue des échanges trans-Manche. Enfin, les maisons circulaires, datée de la fin de La Tène, témoigneraient peut-être de la présence de comptoirs commerciaux mis en place par les populations d'outre-Manche.

The increasingly frequent discovery of roundhouses in North-West France, particularly in Brittany and Normandy, raises many questions concerning the presence of these houses in our regions, given that these structures are well known in British Isles. Underpinned by an inventory of Iron Age roundhouses and a detailed study of their architecture, our aim here is to identify the origin of these constructions, as well as increasing our comprehension of their place in terms of cross-Channel relations. 
Typological comparison indicates that these constructions present numerous similarities with structures in Great Britain. Their location, by the sea and along rivers, and the discovery of artifacts of British imports tend to support the hypothesis of an architectural tradition derived from cross-Channel exchanges. Roundhouses of the end of La Tène period perhaps testify to the presence of trading posts set up by people from across the Channel.

El descubrimiento cada vez más frecuente de casas de forma circular en el suelo francés, en particular en Bretaña y Normandía, es la fuente de muchas preguntas acerca de la presencia de estas habitaciones en nuestra región, mientras que estas estructuras están bien conocidas en las islas británicas. Con un inventario de las casas circulares de la Edad de Hierro y un estudio más completo de su arquitectura, nuestro objetivo es intentar identificar el origen de estas construcciones al mismo tiempo que comprender lo mejor posible su papel dentro de las relaciones entre los dos lados de la Mancha.

Después de una comparación tipológica, parece entonces que estos edificios tienen muchas similitudes con las estructuras del Reino Unido. Su ubicación a lo largo de los ríos y cerca de la costa, así como el descubrimiento de artefactos de importaciones británicas apoyan la hipótesis de una tradición arquitectónica de los intercambios entre los dos lados de la Mancha. Por último, las casas circulares al final de La Tène, testimoniarían tal vez de la presencia de puestos comerciales establecidos por las poblaciones del otro lado de la Mancha.

\section{INDEX}

Palabras claves : Gran Bretaña, Normandía, Edad de Hierro, casas circulares, relaciones entre los dos lados de la Mancha

Keywords : Great Britain, Brittany, Normandy, Iron Age, Roundhouses, Cross-channel relationships

Mots-clés : Grande-Bretagne, Bretagne, Normandie, âge du Fer, maisons circulaires, relations trans-Manche

\section{AUTEUR}

\section{CÉLINE GODARD}

Céline Godard, Archéologue, INRAP Haute-Normandie, 4 boulevard de l'Europe, 14540 Bourguébus. (celine.godard@inrap.fr) 